\title{
SMAD signaling promotes melanoma metastasis independently of phenotype switching
}

\author{
Eylul Tuncer, ${ }^{1}$ Raquel R. Calçada, ${ }^{1}$ Daniel Zingg, ${ }^{1}$ Sandra Varum, ${ }^{1}$ Phil Cheng, ${ }^{2}$ Sandra N. Freiberger, ${ }^{2}$ Chu-Xia Deng, ${ }^{3}$ Ingo Kleiter, ${ }^{4}$ \\ Mitchell P. Levesque, ${ }^{2}$ Reinhard Dummer, ${ }^{2}$ and Lukas Sommer ${ }^{1}$

\begin{abstract}
1Stem Cell Biology, Institute of Anatomy, University of Zurich, Zurich, Switzerland. ${ }^{2}$ Department of Dermatology, University Hospital Zurich, Zurich, Switzerland. ${ }^{3}$ Faculty of Health Sciences, University of Macau, Macau SAR, China. ${ }^{4}$ Department of Neurology, Ruhr-University Bochum, Bochum, Germany and Marianne-Strauß-Klinik, Behandlungszentrum Kempfenhausen für Multiple Sklerose Kranke gGmbH, Berg, Germany.
\end{abstract}

\begin{abstract}
The development of metastatic melanoma is thought to require the dynamic shifting of neoplastic cells between proliferative and invasive phenotypes. Contrary to this conventional "phenotype switching" model, we now show that disease progression can involve malignant melanoma cells simultaneously displaying proliferative and invasive properties. Using a genetic mouse model of melanoma in combination with in vitro analyses of melanoma cell lines, we found that conditional deletion of the downstream signaling molecule Smad4, which abrogates all canonical TCF- $\beta$ signaling, indeed inhibited both tumor growth and metastasis. Conditional deletion of the inhibitory signaling factor Smad7, however, generated cells that are both highly invasive and proliferative, indicating that invasiveness is compatible with a high proliferation rate. In fact, conditional Smad7 deletion led to sustained melanoma growth and at the same time promoted massive metastasis formation, a result consistent with data indicating that low SMAD7 levels in patient tumors are associated with a poor survival. Our findings reveal that modulation of SMAD7 levels can overcome the need for phenotype switching during tumor progression and may thus represent a therapeutic target in metastatic disease.
\end{abstract}

\section{Introduction}

Despite recent progress in therapy, melanoma has remained by far the deadliest skin cancer, with a 5 -year survival rate of only $15 \%$ to $20 \%$ (1). There is increasing evidence that the aggressiveness of the disease is largely due to an intrinsic plasticity of melanoma cells, allowing the dynamic and reversible switching from a high-proliferative/low-invasive to a low-proliferative/high-invasive state $(2-5)$. This so-called "phenotype switching" has been functionally associated with metastasis formation and therapy resistance $(6-8,5)$. Furthermore, phenotype switching has been linked to a shift between a transcriptional program governed by high expression of the microphthalmia-associated transcription factor (MITF), a melanoma lineage-survival oncogene, and a transcriptional program associated with high expression of the receptor tyrosine kinase $\mathrm{AXL}$, a marker for resistance to various targeted therapies (9-12). In contrast to proliferative melanoma cells characterized by high MITF expression, MITF ${ }^{\text {lo }}$ cells with high-invasive properties show a $G_{1}$ cell cycle arrest (13). However, while the distinction between $\mathrm{MITF}^{\mathrm{lo}} \mathrm{AXL}^{\text {hi }}$ and $\mathrm{MITF}^{\text {hi }} \mathrm{AXL}^{\text {lo }}$ phenotypes was used to classify melanomas at the bulk tumor level (14), single-cell RNA-Seq of human melanoma samples has recently led to the identification of a small fraction of double-positive $\mathrm{MITF}^{\mathrm{hi}} \mathrm{AXL}^{\text {hi }}$ cells. It is conceivable that such double-high cells simply represent a transition stage between the 2 phenotypes, but

Conflict of interest: The authors have declared that no conflict of interest exists. Copyright: (5) 2019, American Society for Clinical Investigation.

Submitted: March 30, 2017; Accepted: April 23, 2019; Published: April 30, 2019.

Reference information: / Clin Invest. 2019;129(7):2702-2716.

https://doi.org/10.1172/JCI94295. alternatively, they may also have specific tumorigenic properties that are yet to be elucidated (15).

Phenotype switching has also been linked to tumor progression in several epithelial tumors $(16,17)$. In these cases, a reversible epithelial-to-mesenchymal transition (EMT) promotes invasiveness and stem cell-like features in cancer cells (18), which are driven by a network of embryonic EMT-inducing transcription factors (EMT-TFs) of the SNAIL, TWIST, ZEB, and bHLH/E47 protein families (19). Although melanoma cells are not derived from epithelial cells, they nevertheless show EMT-like processes that can be observed in culture and that have been associated with metastatic spread of the disease (20). In particular, activation of the MAPK pathway by oncogenic BRAF or NRAS promotes a switch from SNAIL2/ZEB2 expression to TWIST1/ZEB1 expression, which enhances invasiveness of melanoma cells (21).

One of the major candidate pathways for driving reversible phenotype switching is SMAD-dependent TGF- $\beta$ superfamily signaling, which controls EMT in many cancers $(22,23)$. Sustained expression of EMT-TFs in breast cancer cells is directly regulated by autocrine TGF- $\beta$ signaling (24). In melanoma, several TGF- $\beta$ isoforms and NODAL as well as different BMP ligands were shown to be expressed by tumor cells and to promote invasiveness in cell culture or in organotypic human skin cultures $(6,7$, $25-27)$. In support of this, attenuation of TGF- $\beta$ signaling by overexpression of SMAD7, an inhibitory SMAD protein (28), or by treatment with a small molecule inhibitor reduced bone metastasis formation from cells injected into the cardiovascular system in immunocompromised mice $(29,30)$.

However, other studies reported that melanoma cells exhibit partial resistance to the antiproliferative activity of TGF- $\beta$ fam- 
ily factors (31). Moreover, TGF- $\beta$-dependent SMAD signaling and transcription were not restricted to invasive cells, but were also observed in proliferative human melanoma cells (31). Likewise, in human tissue samples, nuclear pSMAD2/3, which mediates canonical TGF- $\beta$ signaling, was detected at all stages of the disease, including in benign hyperplastic lesions and cutaneous primary melanoma as well as in invasive melanoma (32). Finally, inhibition of SMAD2/3 signaling by SMAD7 overexpression not only affected the invasiveness of melanoma cells, but also reduced their capacity to grow in vitro and upon transplantation into immunocompromised mice (29).

The combined data suggest that SMAD-mediated signaling may exert various functions in melanoma, which are likely influenced by the cellular context and the TGF- $\beta$ superfamily ligands the tumor cells are exposed to (23). Therefore, in an attempt to mimic the tumor microenvironment with respect to TGF- $\beta$ dependent SMAD signaling, we treated melanoma cells with various combinations of TGF- $\beta$ family factors and addressed the relevance of overall TGF- $\beta /$ SMAD signaling in melanoma in vivo by means of a genetic mouse model, in which tumors develop spontaneously within an undisturbed 3D environment. Searching for factors modulating the proliferative and invasive capacities of melanoma cells, we identified SMAD7 as a repressor of a transcriptional program associated with concomitant MITF $^{\text {hi }}$ AXL $^{\text {hi }}$ expression. In fact, conditional Smad7 deletion in vivo resulted in the emergence of a MITF ${ }^{\text {hi }} \mathrm{AXL}^{\text {hi }}$ subpopulation of cells that were simultaneously proliferating and invasive and associated with increased macrometastasis formation. These experiments identify integrated SMAD signaling as a key driver of melanoma initiation, growth, and metastatic progression, pointing to a new therapeutic vulnerability in melanoma.

\section{Results}

Conditional deletion of Smad4 in the adult melanocytic lineage does not impair proliferation and survival. Downstream of TGF- $\beta$ superfamily signaling, the receptor-associated SMAD (R-SMAD) proteins SMAD2/3 and SMAD1/5/8, are activated by TGF- $\beta /$ ACTIVIN/ NODAL or BMP signals, respectively $(33,34)$. Activated R-SMADs interact with the common partner SMAD4, which is essential for all canonical transcriptional responses (35). To address whether SMAD signaling is essential for homeostasis of normal melanocytes, we injected 6-week-old Tyr::Cre ${ }^{E R T 2} \operatorname{Smad} 4^{f / f l} \mathrm{R} 26 \mathrm{R}-\mathrm{LSL}$ $\mathrm{LacZ}$ mice with tamoxifen (TM) intraperitoneally for 5 days prior to dorsal hair plucking, which induces synchronized hair growth (Supplemental Figure 1, A-C; supplemental material available online with this article; https://doi.org/10.1172/JCI94295DS1). Recombination efficiency was $61 \% \pm 5 \%$, as assessed by counting the percentages of recombined, $\beta$-gal-positive hair follicles (Supplemental Figure 1D). Unlike in control animals, TM-induced conditional $\mathrm{KO}$ (cKO) of Smad4 resulted in hypopigmentation of a subset of regenerating hairs (Supplemental Figure 1, E and F). However, this pigmentation defect was not associated with a reduction in the number of hair bulbs containing recombined melanocytes ( $\beta$-gal/Dct double-positive) between control (Tyr::Cre ${ }^{E R T 2} R 26 R$ LSL-LacZ) and cKO (Tyr::Cre ${ }^{E R T 2} S m a d 4^{f / f l}$ R26R-LSL-LacZ) animals upon plucking (Supplemental Figure 1, F and G). Additionally, the number of recombined melanocytes per hair bulb and their proliferation rate was comparable between control and cKO mice (Supplemental Figure 1, G-I). In support of these results, SMAD4 knockdown in human melanocytes also resulted in impaired pigmentation and decreased expression of melanocyte differentiation genes, such as TYRP1, TYRA, and MC1A, while proliferation rates were unchanged upon SMAD4 inactivation (Supplemental Figure $1, \mathrm{~J}-\mathrm{L})$. The combined data suggest that Smad4 is not required for adult melanocyte survival and proliferation, although it is required for normal pigmentation.

Loss of Smad4 prevents tumorigenesis in a genetic mouse model of melanoma. To investigate the function of TGF- $\beta$ signaling in melanoma, we used genetically engineered mice that harbor a Tyr::Nras ${ }^{\mathrm{Q} 61 \mathrm{~K}}$ transgene in combination with Cdkn4a $\left(p 16^{\text {Ink4a }}\right)$

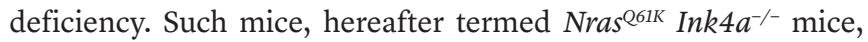
develop hyperplastic lesions marked by ectopic dermal pigmentation and spontaneously form melanomas (36). These were bred with Tyr::Cre ${ }^{E R T 2}$, Smad4 $4^{f / f l}$ mice to eventually yield Smad $4^{f / f l}$ $\mathrm{Nras}^{\mathrm{Q} 61 \mathrm{~K}} \mathrm{Ink} 4 \mathrm{a}^{-/-}$offspring, in which TM treatment leads to melanocyte-specific conditional Smad4 ablation in cells marked by expression of $\beta$-gal (Figure 1A). TM treatment was done at 1 month of age, that is, before control mice develop detectable melanomas (Figure 1B). As long as Smad4 remained intact (Smad $4^{f l / f l}$, no $\mathrm{TM}$; or Smad4 $4^{f / f l}+\mathrm{TM}$, but in the absence of $\left.C r e^{E R T 2}\right), 76 \% \pm 5 \%$ of hair follicles were associated with ectopic pigmentation. Two months after TM injection, the recombination efficiency in such hyperplastic lesions was $66 \% \pm 6 \%$, as assessed by counting $\beta$-gal/ Pax3 double-positive melanocytic cells. Conditional loss of Smad4 resulted in a marked decrease of hyperplastic lesions, with only $13 \% \pm 3 \%$ of hair follicles displaying ectopic dermal pigmentation (Figure 1, C and D). In these mice, melanocytic, Pax3-positive cells were positive for $\beta$-gal, suggesting that Smad4 is not essential for the survival of premalignant melanocytic cells (Figure 1, E and F). Importantly, the loss of Smad4 was associated with a significant decrease in the number of $\beta$-gal-positive skin melanomas (diameter $\geq 2 \mathrm{~mm}$ ), which readily emerged after approximately 5 months of age in corresponding control mice (Figure 1, G and H). Consistent with the decreased skin tumor load, Smad4-cKO mice displayed strongly reduced numbers of metastatic lung nodules as compared with control animals (Figure 1I). Consequently, overall as well as melanoma-specific survival was highly increased in Smad4-cKO mice (Figure 1, J and K). These findings reveal a requirement for Smad4 in melanoma formation.

Loss of Smad4 leads to decreased proliferation in established skin tumors. To address the cellular mechanism mediated by Smad4-dependent signaling in established melanoma, we conditionally deleted Smad4 by TM treatment after the appearance of visible tumors (Figure 2A). The phenotype of Smad4 heterozygous animals did not differ from that of Smad4 WT mice. Hence, Nras $^{Q 61 K}$ Ink4 $^{-/-}$Tyr::Cre ERT2 Smad4 ${ }^{f / W T}$ R26R-LSL-LacZ animals were used as control animals for further analyses. High recombination efficiency was apparent at the day of sacrifice (Figure 2B). While apoptosis in recombined melanoma cells was generally low in Smad4-cKO mice and comparable to levels seen in control melanoma tissue, proliferation was significantly reduced in cKO mice, as demonstrated by labeling of $\beta$-gal-positive recombined cells with the proliferation marker Ki67 (Figure 2, C-E). Thus, while Smad4 has no apparent effect on proliferation of normal 
A

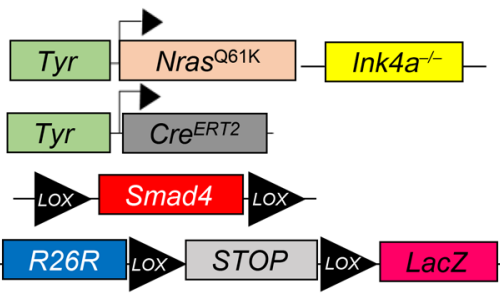

B

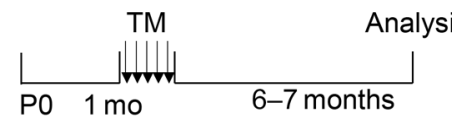

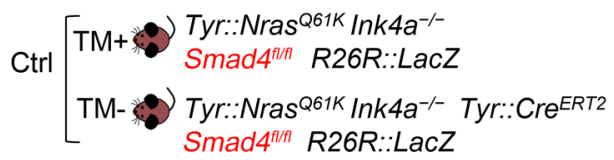

$\mathrm{cKO}\left[\mathrm{TM}+\mathrm{Tyr}:: \mathrm{Nras}^{\mathrm{Q61K}} \operatorname{Ink} 4 \mathrm{a}^{-/-}\right.$Tyr::Cre $\mathrm{CRT2}^{\mathrm{E}}$ Smad4 ${ }^{\mathrm{fl} / \mathrm{fl}}$ R26R::LacZ

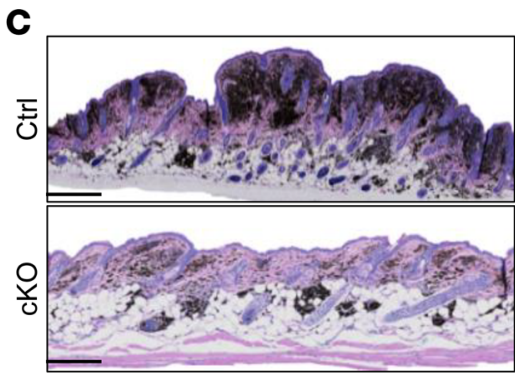

E Hyperplasia

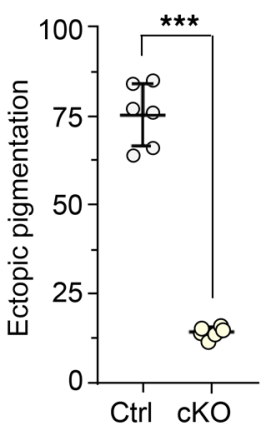

F

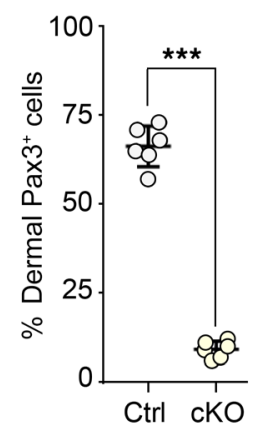

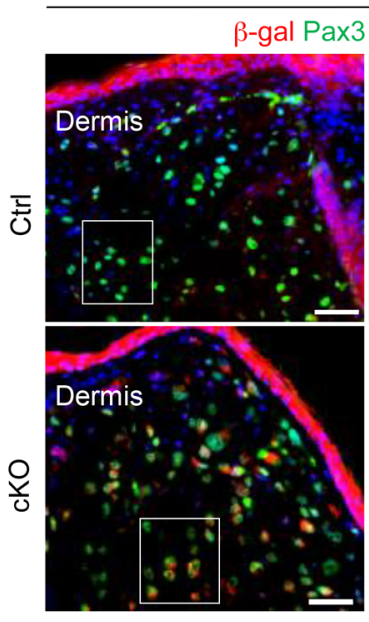

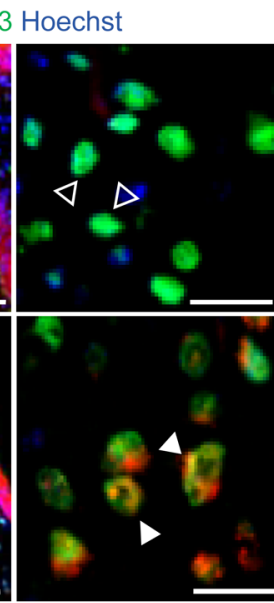

G

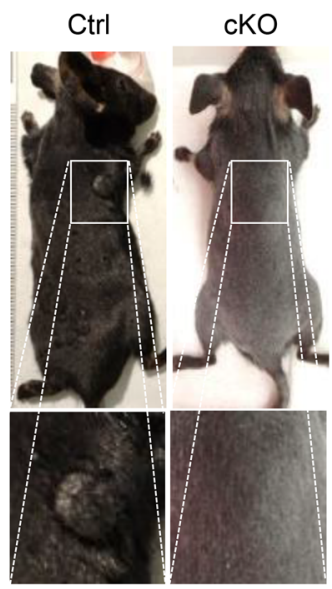

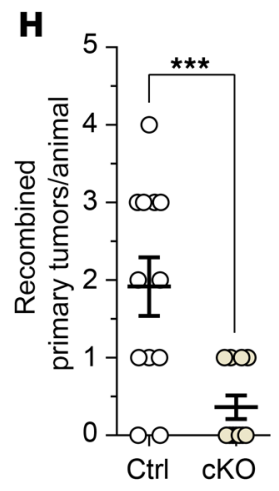

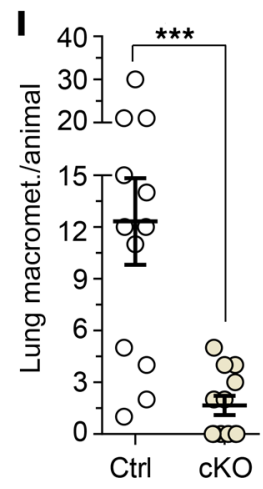

J

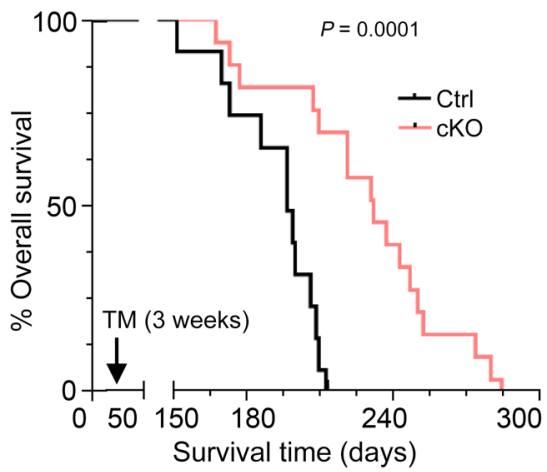

K

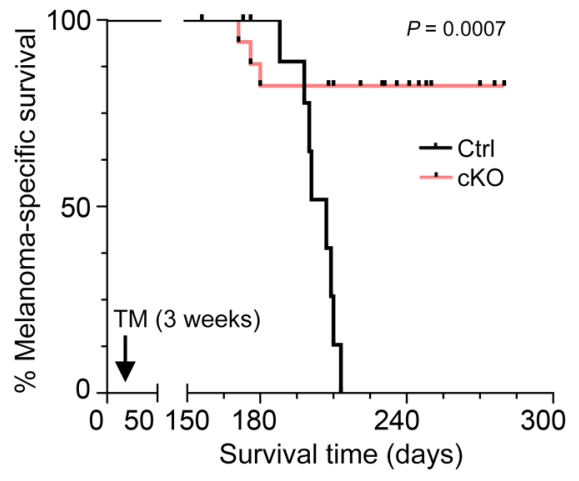

Figure 1. Conditional Smad4 deletion in a genetic mouse model of melanoma prevents tumorigenesis. (A) Schematic of the melanoma mouse model harboring Tyr::Nras ${ }^{061 \%}$, Ink4a ${ }^{-1}$, Tyr::Cre ${ }^{E R T 2}$, floxed Smad4, and R26R Cre-reporter alleles. (B) Experimental strategy used to analyze early loss of Smad4 before appearance of visible melanomas. Control mice either lacked the Tyr::Cre ERT2 allele or were not treated with TM. (C) Representative H\&E staining of trunk skin sections of control and cKO mice at day of sacrifice showing ectopic dermal hyperpigmentation. (D) Quantification of the percentage of hair follicles exhibiting ectopic pigmentation in control (nontreated with TM) and cKO mice ( $n=350$ hair follicles quantified from 6 different mice). (E) Immunofluorescent staining for $\mathrm{Pax3}$ (control, nontreated with TM) and Pax3+ $\beta$-Gal+ $($ cKO) on back skin sections at 6 months to quantify extent of dermal hyperplasia. Open arrowheads indicate Pax3 $3^{+}$cells, white arrowheads Pax3 ${ }^{+} \beta$-Gal ${ }^{+}$cells. (F) Quantification of the percentage of dermal Pax3 $3^{+}$cells between hair follicles $(n=300$ hair follicles from 6 different cKO and control mice). (C) Macroscopic pictures of a control and a Smad4-cKO littermate 6 months after Smad4 ablation. (H) Quantification of recombined primary tumor numbers per control $(n=12)$ and cKO $(n=11)$ mice at the day of sacrifice. (I) Quantification of lung macrometastases counts at day of sacrifice using macroscopic pictures and staining on sections $(n=12)$. ( $\mathbf{J}$ and $\mathbf{K})$ Kaplan-Meier curves displaying overall and melanomaspecific survival, respectively, of control $(n=12)$ and Smad4-cKO $(n=17)$ animals. Vertical bars (K) indicate mice censored because of melanoma-unrelated tumors developing due to constitutive loss of Ink4a. Data are represented as a mean of 3 independent experiments \pm SEM (H and $\mathbf{I})$ and \pm SD ( $\mathbf{D}$ and $\mathbf{F})$. ${ }^{* * *} P<0.001$, unpaired Student's $t$ test (D, F, H, I), log-rank Mantel-Cox test (J and K). Ctrl, control; HF, hair follicle. Scale bars: $50 \mu \mathrm{m}$ (E); $500 \mu \mathrm{m}$ (C).

cells (Supplemental Figure 1, H, I and K), it controls proliferation of melanoma cells, indicating that the combined action of TGF- $\beta$ superfamily members promotes, rather than counteracts, tumor growth in vivo.
To clarify the mechanism underlying Smad4-dependent cell cycle control, we determined changes in the expression of several cyclin-dependent protein kinase (CDK) inhibitors after siRNAmediated Smad4 silencing in melanoma cell lines derived from 
A

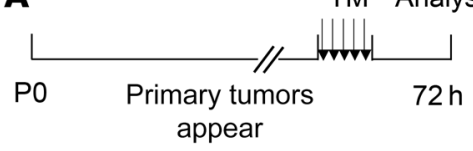

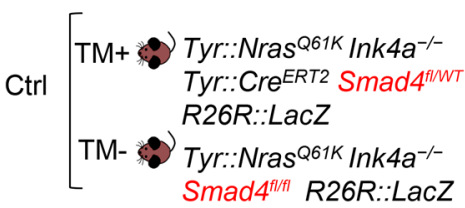

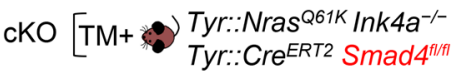
R26R:LacZ

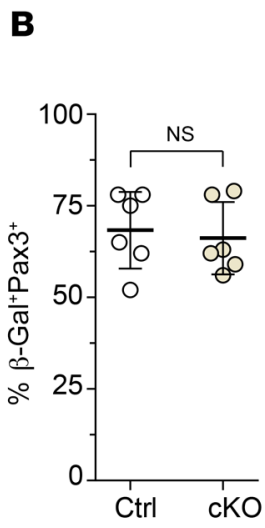

C
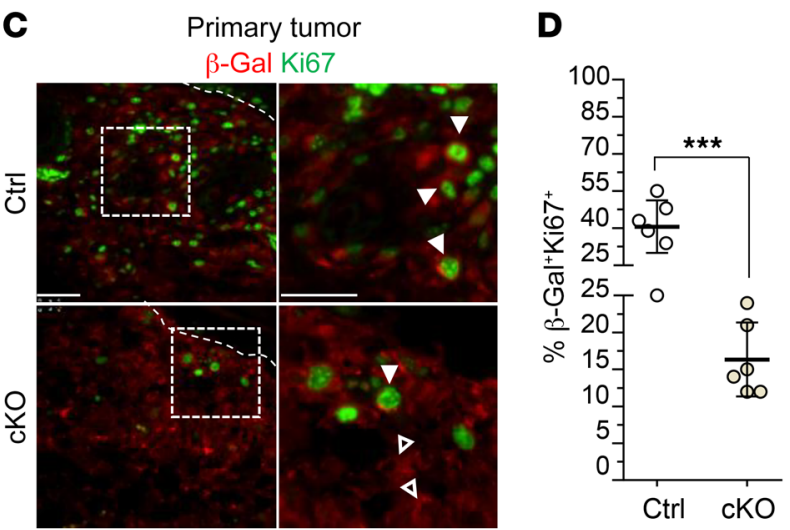

D
E

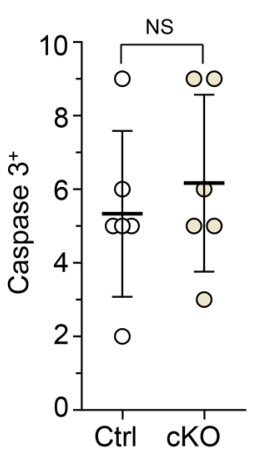

F

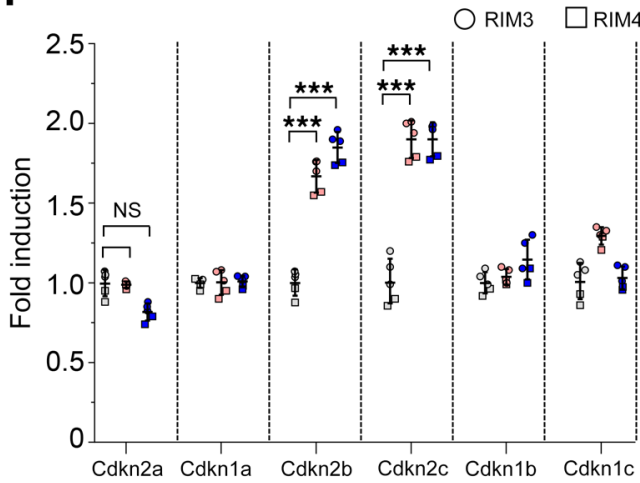

G

G RIM3
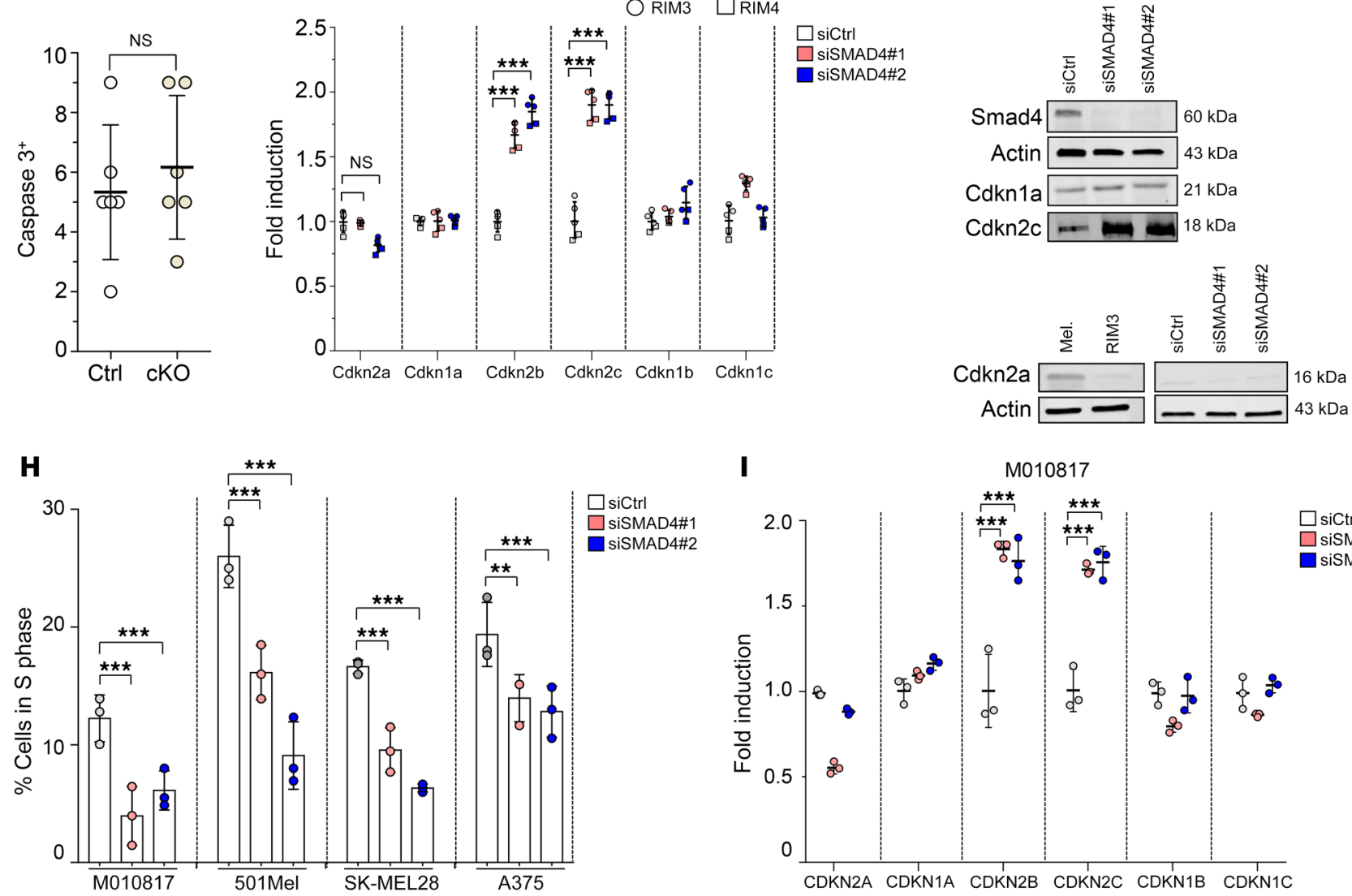

Figure 2. Loss of Smad4 leads to decreased proliferation in established skin tumors. (A) Experimental strategy used to analyze the deletion of Smad4

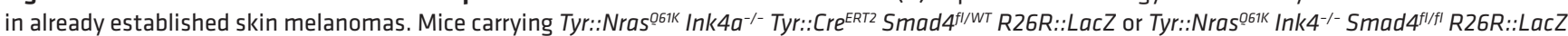
were used as controls. (B) Recombination efficiency was calculated by counting percentages of $\beta$-gal'Pax $3^{+}$cells on primary tumor sections ( $n=6$, cKO and

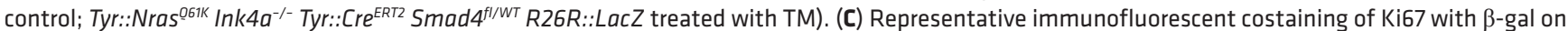
skin melanoma sections 72 hours after conditional deletion of Smad4. White arrowheads indicate Ki67 ${ }^{+} \beta$-Gal ${ }^{+}$cells; open arrowheads indicate Ki67 $\beta \beta$-Gal ${ }^{+}$

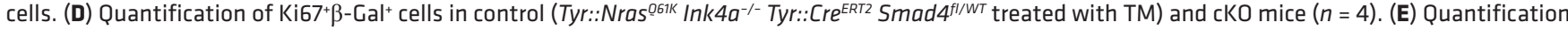
of apoptotic cells by IHC for activated caspase-3 on skin sections of control and cKO mice $(n=4)$. (F) Quantitative reverse transcription PCR (RT-qPCR) for expression of multiple $G_{1}$ cell cycle inhibitors in RIM3 $(n=3)$ and RIM4 $(n=2)$ cell lines after siRNA treatment. (G) Western blot performed on nuclear extracts from RIM3 for cell cycle regulator Cdkn1a (p21 War1), Cdkn2c (p18 $\left.{ }^{\operatorname{lnk} 4 c}\right)$, and Cdkn2a (p16 $\left.{ }^{\operatorname{lnk} 4 a}\right)$ protein expression. (H) Percentage of S phase cells upon SMAD4 knockdown in various human melanoma cell lines. (I) RT-qPCR analysis of the same cell cycle regulators in M010817 human melanoma cell line. Data are represented as the mean \pm SEM (D) and \pm SD (B, E, F, H, I). ${ }^{* *} P<0.01$; ${ }^{* *} P<0.001$. RT-qPCR results are shown as mean \pm SD of 3 biological replicates. For Western blots, representative examples are shown. $P$ values calculated with unpaired Student's $t$ test (B, D, E, F, H, I). Mel, Melan-A mouse melanocyte line. Scale bar: $50 \mu \mathrm{m}$. 
A

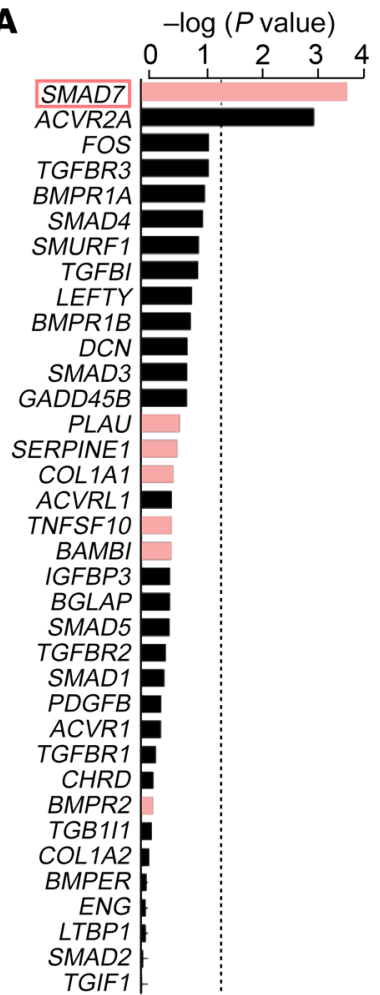

B

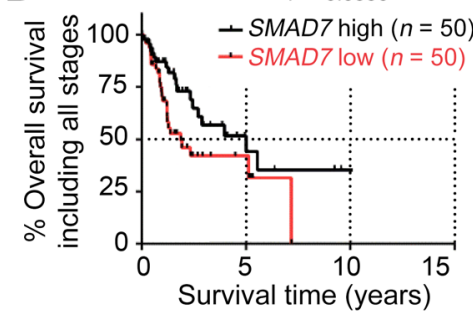

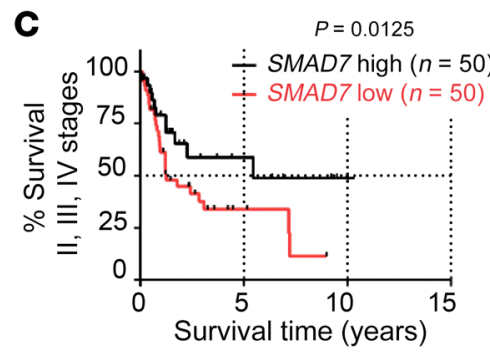

D

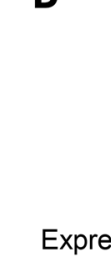

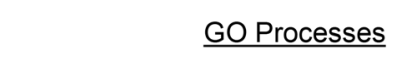

Integrin-mediated cell adhesion and migration Upregulation of MITF in melanoma TGF, WNT and cytoskeletal remodeling Chemokines and adhesion Regulation of epithelial-to-mesenchymal transition (EMT) ECM remodelling Oligodendrocyte differentiation from adult stem cells Elevation of intraocular pressure as glaucoma factor

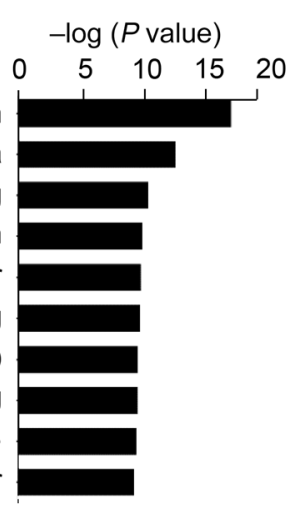

Figure 3. Low SMAD7 levels are associated with poor prognosis in human melanoma patients. (A) $P$ values are given for Kaplan-Meier analysis comparing percentage of overall survival of melanoma patient cohorts $(n=454)$ based on TCGA data for 36 identified transcripts of TCF- $\beta$ /BMP pathway components. For each gene, low/high expression levels were based on transcript levels found in the bottom and top 50 patients, respectively. Red bars show that lower levels of the transcripts correlate with poor survival. whereas black bars indicate that higher levels of the transcript correlate with poor survival. (B and C) Kaplan-Meier curves comparing overall percentage survival and patient survival rate in stages II, III, and VI with respect to SMAD7 transcript levels based on 454 patients. (D) Significantly changed top 10-ranking GeneGo process networks associated with low/high SMAD7 expression based on MetaCore Database.

Nras ${ }^{\text {Q61K In }}{ }^{2} 4 a^{-1-}$ mice (RIMs) (37). Of all CDK inhibitors tested, Cdkn2b (p15 $\left.{ }^{\text {Ink4b }}\right)$ and Cdkn2c (p18 $\left.{ }^{\text {Ink4c }}\right)$ were significantly upregulated in Smad4-knockdown cells (Figure 2, F and G).

To address whether SMAD4 regulates proliferation also in human melanoma cells, we performed SMAD 4 knockdown experiments in various human melanoma cell lines. Of note, in all cell lines tested, the percentages of cells in the S-phase were decreased after siSMAD4 transfection relative to siControl transfection, likely because downregulation of SMAD4 expression counteracted cell cycle progression by repressing $G_{1} / S$ transition (Figure $2 \mathrm{H}$ and data not shown). Importantly, in data that were analogous to those obtained with murine melanoma cells, cell cycle arrest induced by SMAD4 inactivation was associated with increased expression of CDKN2B (p15 $\left.5^{\mathrm{INK} 4 \mathrm{~b}}\right)$ and CDKN2C (p18 ${ }^{\mathrm{INK} 4 \mathrm{c}}$ ), while the expression of other CDK inhibitors was not altered (Figure 2I and Supplemental Figure 2, A-D).Thus, both in mouse and human melanoma cells, SMAD4-mediated signaling appears to control proliferation by suppressing expression of the respective CDK inhibitors.

BMP7 signaling promotes melanoma cell growth. Until now, members of the TGF- $\beta$ superfamily have been associated with cytostatic and/or proinvasive functions in melanoma cells (38). However, our in vivo data indicate that one or more factors that signal through Smad4 must have a proproliferative role. Most of the TGF- $\beta$ family ligands known to elicit responses in human mel- anoma cells are expressed by the tumor cells themselves, where they induce their own expression through a positive amplification loop (27, 39-41). Likewise, several TGF- $\beta$ superfamily members, notably TGF- $\beta 2$ and BMP7, were concomitantly expressed by tumors derived from $\mathrm{Nras}^{\mathrm{Q}}{ }^{\mathrm{G} / \mathrm{IK}} \mathrm{In} \mathrm{k} 4 \mathrm{a}^{-/-}$mice (Supplemental Figure 3A). Thus, we functionally investigated these factors to identify ligands potentially responsible for melanoma cell proliferation. Cell cycle analysis of 2 cell lines (M010817 and 501Mel) revealed that, among the factors analyzed, BMP7 induced cell cycle progression. The percentages of cells in the S-phase were significantly increased from, respectively, $18 \pm 1 \%$ and $18 \% \pm 2 \%$ in control to $28 \% \pm 3 \%$ and $27 \% \pm 2 \%$ after exposure to BMP7 (Supplemental Figure 3, B and C). In contrast, the other TGF- $\beta$ superfamily factors tested suppressed proliferation in human melanoma cells, as previously reported (Supplemental Figure 3, B and C) $(6,27,42)$. In agreement with these earlier reports, human melanoma cells exposed to TGF- $\beta 2$, NODAL, or BMP4 also changed their morphology and exhibited a decreased cell-substrate adherence while BMP7 did not (Supplemental Figure 3D).

To substantiate the above findings, we correlated expression levels of BMP7 and TGFB2 with RNA-Seq data obtained from various proliferative and invasive human melanoma cell lines in culture. Among others, TGFB2 mRNA was robustly expressed in patient-derived cell cultures representing an invasive melano- 
ma phenotype (WNT5A $A^{h i}, A X L^{h i}, Z E B 1^{h i}, S O X 9^{h i}$ ) (Supplemental Figure $3 \mathrm{E}$ ). In contrast, markers for proliferative melanoma cells $\left(S O X 10^{h i}, M_{T T}{ }^{h i}\right)$ correlated with BMP7 expression levels (Supplemental Figure 3E).

Since activation of R-SMADs is required for canonical TGF- $\beta$ signaling, we investigated whether proliferative versus invasive ligands preferentially signal through specific SMADs. Western blot analysis for pSMAD1/5/8 and pSMAD2/3 (indicators of BMP and TGF- $\beta$ signaling activity, respectively) verified signaling specificity between different TGF- $\beta$ superfamily members in human melanoma cells (Supplemental Figure 3, F-H). Remarkably, both BMP4 and BMP7 signaled through SMAD1/5/8 phosphorylation, although BMP4 counteracted proliferation and induced an invasive program when added to melanoma cells, whereas BMP7 increased the percentage of cells in the S phase (Supplemental Figure $3, \mathrm{~B}-\mathrm{D}$ and $\mathrm{H}$ ). Thus, the distinct biological activities of TGF- $\beta$ superfamily members cannot simply be explained by differential usage of the known canonical SMAD signaling pathways.

Loss of SMAD7 is clinically relevant and associated with a MITF $F^{\text {hi }}$ $A X L^{h i}$ transcriptional state. Our data show that certain TGF- $\beta$ superfamily factors expressed in melanoma display opposite effects on cell growth and invasiveness. In an attempt to identify modulators and effectors of TGF- $\beta$ /SMAD signaling possibly relevant for melanoma progression, we first examined RNA-Seq and clinical data from the skin cutaneous melanoma dataset of The Cancer Genome Atlas (TCGA-SKCM) (43). Survival analysis of TCGA-SKCM showed that, among 36 SMAD signaling components, the inhibitory SMAD protein SMAD7 stood out as the clinically most relevant factor (Figure 3A). In fact, patients with low SMAD7 transcript levels had significantly shorter overall survival as compared with those having high SMAD7 transcripts levels $(P=0.0356)$ (Figure $3 \mathrm{~B})$. The SMAD7 $7^{\text {hi }}$ and SMAD7lo patient cohorts did not exhibit obvious differences with respect to clinically relevant $B R A F$ and NRAS mutations (data not shown). The difference in survival was even more evident when comparing among the SMAD7 $7^{\text {lo }}$ and SMAD7 ${ }^{\text {hi }}$ groups those patients that displayed advanced stage disease at the time of analysis (stages II-IV) $(P=0.0125)$ (Figure 3C). Furthermore, gene ontology (GO) analysis using differential gene signatures of SMAD7 $7^{\text {lo }}$ and SMAD7 ${ }^{\text {hi }}$ patients revealed that categories crucial for melanoma biology, such as regulation of EMT, cell adhesion and migration, and cytoskeletal remodeling as well as upregulation of MITF, were significantly enriched (Figure 3D). Finally, we performed immunofluorescent staining for SMAD7 and the human melanoma marker S100A on 13 different patient samples and quantified the percentage of SMAD7/S100A double-positive cells at superficial (upper layer of epidermis, upper nodules) versus deep sites (deeper nodules and dermis) of primary melanoma samples (Supplemental Figure 4, A and B). While SMAD7 was strongly expressed in superficial regions of melanoma nodules, its expression was significantly weaker or absent in deeper areas and in dermal invasive melanoma cells, suggesting that $\mathrm{SMAD}^{\text {lo }}$ cells may be predominantly associated with the invading front of human melanoma.

To address the physiological role of SMAD7 and its potential association with TGF- $\beta$ signaling in melanoma cells, we first reduced SMAD7 levels using siRNAs. This led to a significant increase in the levels of both pSMAD2/3 and pSMAD1/5/8, demonstrating the capacity of SMAD7 to inhibit canonical TGF- $\beta$ signaling in melanoma cells (Supplemental Figure 4, C-E). To characterize the molecular pathways regulated by SMAD7-mediated signaling in melanoma, M010817 cells treated with siSMAD7 or siControl were subjected to RNA-Seq. Similarly to the TCGA patient analysis (Figure 3D), the GO analysis by ClueGO demonstrated significant enrichments in pathways associated with cell cycle core components and cell-cell adhesion (Figure 4, A and B, Supplemental Figure 4F, and Supplemental Table 1) (44). Specifically, a comprehensive EMT program, including cell-cell adhesion molecules and transcriptional regulators associated earlier with a melanoma invasive gene program (45) was upregulated upon knockdown of SMAD7 (Figure 4, C and D, and Supplemental Table 2). In addition, however, there was also a significant overlap between SMAD7-dependent transcriptional changes and a previously described proliferative melanoma gene signature (45) (Figure 4, C and D, and Supplemental Table 3).

An increase in the expression of EMT regulators has been reported to anticorrelate with an $\mathrm{MITF}^{\text {hi }}$ gene expression program $(14,46)$. Moreover, the ratio of MITF/AXL expression has been used to define proliferative versus invasive behaviors of melanoma cells $(3,14)$. Therefore, we next assessed which of the genes that constitute the published MITF ${ }^{\text {hi }}$ and $\mathrm{AXL}^{\text {hi }}$ programs were differentially expressed in the TCGA-SKCM-based $S M A D 7^{l o}$ patient group and in $S M A D 7$-silenced human melanoma cells, respectively (13). Surprisingly, genes of both the MITF and the AXL program were mostly upregulated in $\mathrm{SMAD} 7^{\mathrm{lo}}$ patient melanomas or upon loss of SMAD7 in melanoma cells (Figure 4, E-H, Supplemental Table 4). These data suggest that melanoma cells with decreased SMAD7 levels exhibit a MITF ${ }^{\text {hi }}$ AXL $^{\text {hi }}$ transcriptional state.

Loss of SMAD7 boosts proinvasive TGF- $\beta / N O D A L$ signaling in the presence of proproliferative BMP7. According to our gene expression analysis, SMAD7 appears to be implicated in both cell cycle and cell adhesion/EMT control in melanoma. To functionally test this hypothesis, we assayed the effect of siRNA-mediated SMAD7 knockdown in the context of combinatorial TGF- $\beta$ superfamily signaling. Control experiments with SMAD7 WT cells showed that individual TGF- $\beta 2$ or NODAL treatment reduced proliferation and cell-substrate adhesiveness of human melanoma cells, which was accompanied by increased invasiveness in a Matrigel Transwell assay and upregulation of an EMT gene expression signature characteristic for invasive melanoma (Figure 5, Supplemental Figure 5, and Supplemental Figure 6). In contrast, BMP7 stimulated proliferation, prevented cell detachment from the substrate, and suppressed the EMT signature even when added together with TGF- $\beta 2 /$ NODAL (Figure 5, A-D and G, Supplemental Figure 5, and Supplemental Figure 6). Thus, in the presence of TGF- $\beta 2 /$ NODAL, proproliferative BMP7 is dominant and able to override the cytostatic effects of TGF- $\beta 2 /$ NODAL in human melanoma cells.

We next performed similar cell cycle analyses and invasion assays in the context of SMAD7 knockdown. Although downregulation of SMAD7 did not alter the cell cycle-promoting effect of BMP7 in human melanoma cells, SMAD7 knockdown in the presence of BMP7 reversed the cell cycle arrest observed after TGF- $\beta 2$ treatment, hence rendering treatment with TGF- $\beta 2$ compatible with cell proliferation (Figure 5, A-C, and Supplemental Figure 5, $\mathrm{A}-\mathrm{C})$. In contrast to the capacity of BMP7 to override the cytostat- 
A

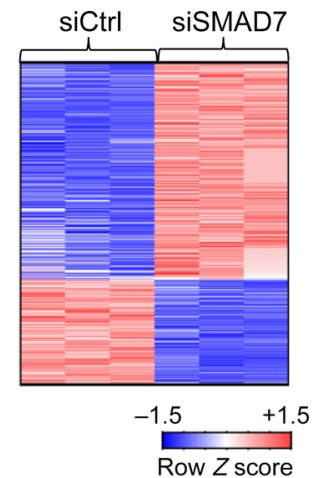

B
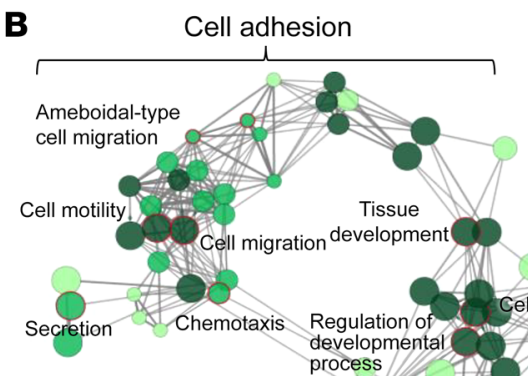

Cell cycle

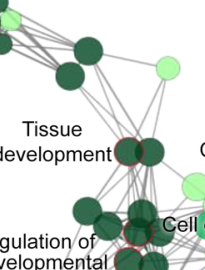
Regulation of
developmental
process process

Row $Z$ score

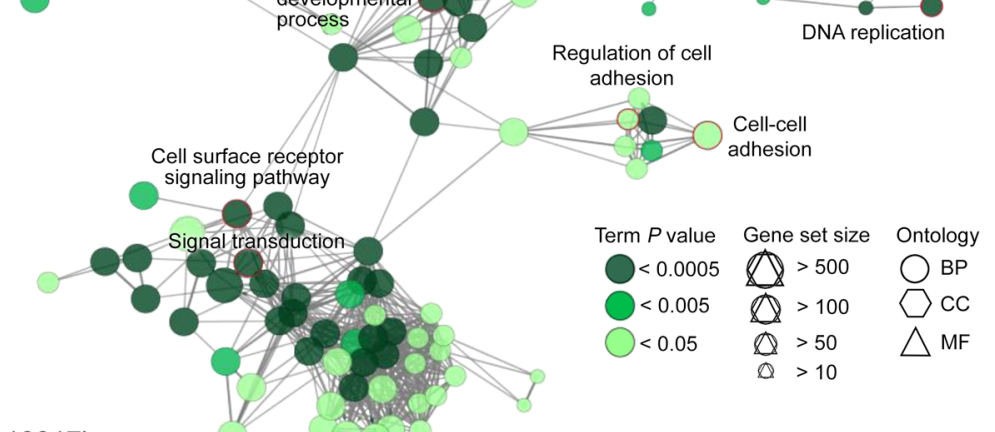

SMAD7 (RNA-seq M010817)

inv. program

Proliferative program

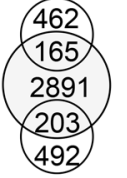
으 Invasive program

Verfaillie pro. program

E

MITF program

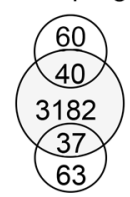

$\mathbf{F}$

Low SMAD7 vs. high SMAD7 (TCGA)

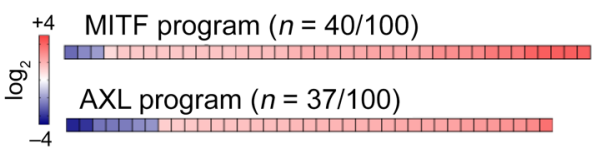

AXL program
G MITF program

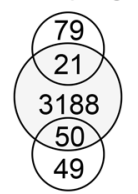

H

AXL program

Figure 4. Low SMAD7 levels are associated with altered cell adhesion and cell cycle programs in human melanoma cell lines. (A) Heatmap showing genes differentially expressed in SMAD7 knockdown and control M010817 cells (3557 genes, row z-score from 3 replicate RNA-Seq experiments). The gene list was generated using a 1.5-fold-change cut-off, and a $P$ value of 0.05 . Of these, 1586 were upregulated and 1971 downregulated. (B) GO analysis based on differentially regulated genes upon SMAD7 knockdown. Each individual node shows an enriched G0 term $(P<0.05)$ (Corrected with Bonferroni's step down procedure). BP, biological process; MF, molecular function; CC, cellular component. A fully labeled version is given in Supplemental Figure 4F. (C) Venn diagram shows the upregulated and downregulated common genes involved in previously described Verfaillie invasive (Inv.) and proliferative (Pro.) program. (D) Heatmap of differentially expressed genes associated with Verfaillie invasive and proliferative programs (proliferative program, $n=165 / 627$, invasive program. $n=203 / 695$ ) (44) Red, increased; blue, decreased expression. ( $\mathbf{E}$ and $\mathbf{G}$ ) Venn diagram showing the overlap between MITFi and AXL ${ }^{\text {hi }}$ gene expression programs derived from patient melanoma samples obtained by single-cell RNA sequencing (16) with genes changed between low/high expressing SMAD7 patients or upon siRNA-mediated knockdown of SMAD7 in M010817. (F and $\mathbf{H}$ ) Heatmaps indicate differentially expressed MITF and AXL program genes, respectively. For gene lists corresponding to $\mathbf{D}, \mathbf{F}$, and $\mathbf{H}$, see Supplemental Tables 3 and 4 , respectively.

ic effect of TGF- $\beta 2$, however, BMP7 treatment only partially counteracted the loss-of-adhesion phenotype mediated by TGF- $\beta 2 /$ siSMAD7. Indeed, in BMP7/TGF- $\beta 2 /$ siSMAD7-treated cultures, there was still a significant increase in the number of cells in suspension as compared with in control cultures (Figure 5D and Supplemental Figure 5D). Consistent with these observations, siSMAD7 treatment of cells concomitantly exposed to TGF- $\beta 2$ and BMP7 restored an EMT gene expression signature characteristic of invasive melanoma cells (Figure 5G) and increased invasion in Matrigel, thereby reinforcing the proinvasive properties of TGF- $\beta 2$ (Figure 5, $\mathrm{E}$ and F, and Supplemental Figure 5E). Similar results were observed upon combinatorial treatment with NODAL. While BMP7/NODALtreated cells had a noninvasive phenotype, BMP7/NODAL/ siSMAD7-treated cells maintained their proliferative capacity, but displayed reduced cell adhesion and an EMT signature reminiscent of invasive cells (Supplemental Figure 6). Thus, reducing SMAD7 levels does not alter proliferation in vitro, but boosts the proinvasive activity of TGF- $\beta 2 /$ NODAL even in the presence of BMP7.

To substantiate that these cells retain the capacity to proliferate and be invasive, we pulsed human melanoma cells with the thymidine analogue EdU after 48 hours of silencing SMAD7 in the presence of BMP7 and TGF- $\beta 2$ (Figure 6A). Furthermore, we 
A

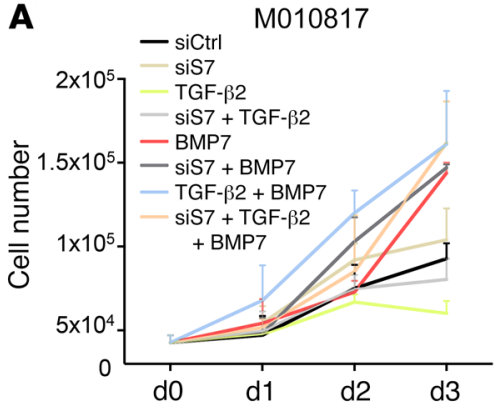

D

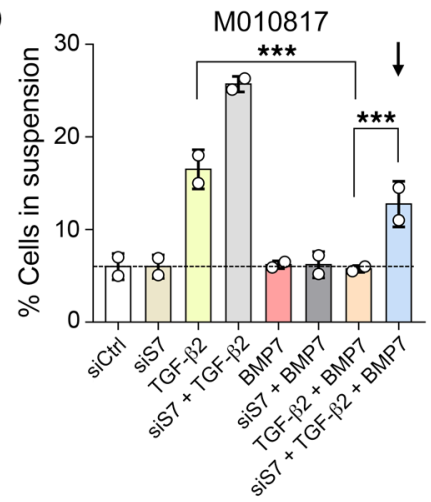

B

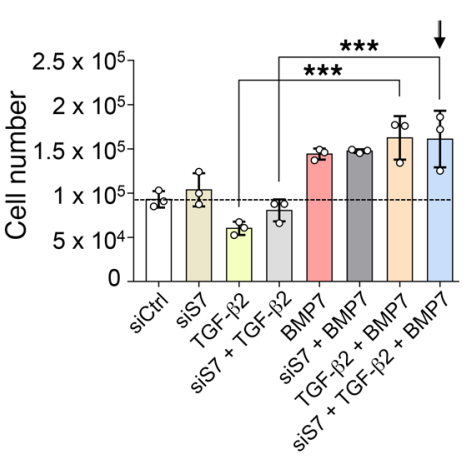

E $\quad$ M010817

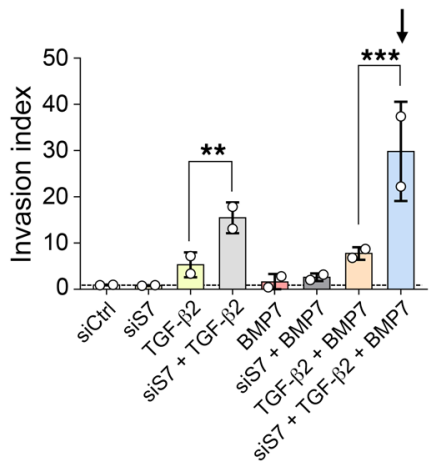

C

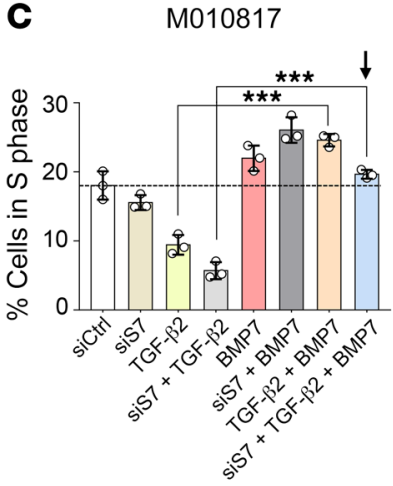

$\mathbf{F}$
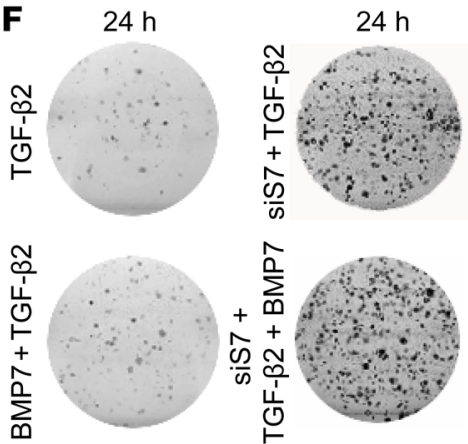

G

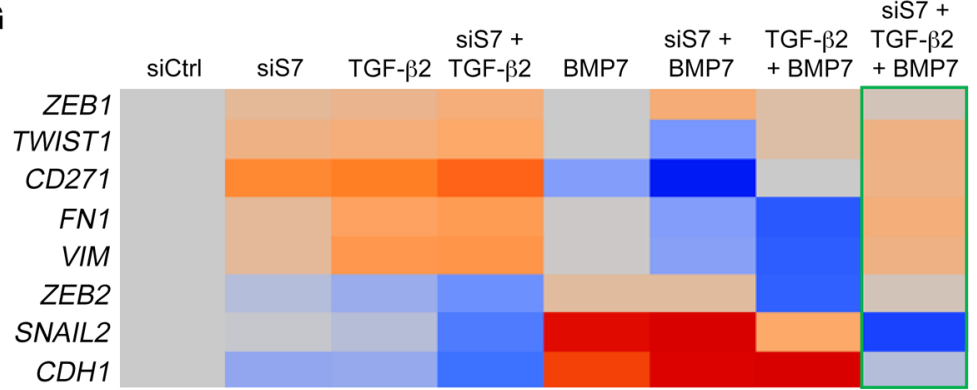

$\operatorname{siS7}+$ fold change
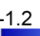
$+1.2$

Figure 5. Loss of Smad7 boosts proinvasive TGF- $\boldsymbol{\beta}$ /NODAL signaling in the presence of proproliferative BMP7. (A) Growth curves of M010817 cells treated either with TCF- $\beta 2$ (10 $\mathrm{ng} / \mu \mathrm{l})$, BMP7 (100 $\mathrm{ng} / \mu \mathrm{l})$, or both, with or without siRNA-mediated SMAD7 knockdown (siS7) ( $n=1$ independent experiment and 3 technical replicates). (B) Bar plots show the number of cells at day 3 after various treatments ( $n=1$ independent experiment and 3 technical replicates). (C) Percentage of S phase cells measured 3 days after ligand treatment based on propidium iodide and EdU staining. Treatment with BMP7 resulted in escape of cells from the TGF- $\beta 2$-mediated cell cycle arrest and increased proliferation. ( $n=3$ independent experiments). (D) Quantification of substrate adherence capacity of M010817 cells upon different treatments. TGF- $\beta 2$-mediated cell detachment was suppressed by BMP7. The percentage of BMP7/TGF- $\beta 2$ / siSMAD7-treated cells in suspension increased as compared with BMP7/TGF- $\beta 2$-treated cells ( $n=2$ independent experiments). (E) Matrigel-based invasion assays of siControl and siSMAD7-depleted M010817 cells with combinatorial treatments. After invading cells had been counted in 5 random microscopic fields in each assay, the results were normalized and are presented as an invasion index ( $n=2$ independent experiments). (F) Photographs of cells invading the membrane, stained with Hoechst. (G) Heatmap shows qRT-PCR analysis for selected EMT genes under ligand treatments ( $n=2$ independent experiments and 3 technical replicates). The gene expression levels are represented as fold-change values transformed to log format compared with control. Color represents expression after normalization to nontreated control cells. Data are represented as a mean of 3 (C), 2 ( $\mathbf{D}$ and $\mathbf{E})$, or 1 (A and B) independent experiments $\pm \mathrm{SD} .{ }^{* *} P<0.01 ;{ }^{* *} P<0.001$, 1-way ANOVA followed by Tukey's multiple comparison test.

tested to determine whether proliferative cells expressed ZEB1, which is characteristic of invasive melanoma cells (21). Strikingly, double staining revealed that BMP7/TGF- $\beta 2 /$ siSMAD7-treated cultures contained significantly higher numbers of EdU and ZEB1 double-positive cells $(23 \% \pm 3 \%)$ in comparison with BMP7/ TGF- $\beta 2$-treated $(9 \% \pm 2 \%)$ or untreated control cultures $(4 \% \pm$ 1\%) (Figure 6, B and C).
Deregulated TGF- $\beta /$ SMAD signaling can alter MITF expression depending on the melanoma cell line (47). Moreover, the ratio of MITF/AXL expression levels has been used to distinguish proliferative and invasive melanoma cells $(3,14)$. Therefore, we also assessed the percentages of cells expressing high versus low levels of MITF, together with AXL expression, upon combinatorial TGF- $\beta$ superfamily treatment (Figure 6, B and D). In cultures left 
A

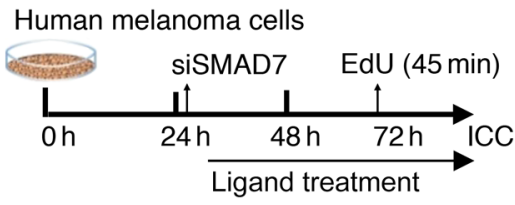

B

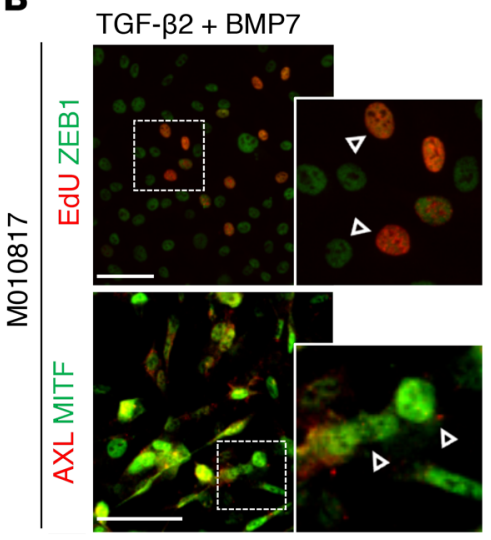

TGF-B2 + BMP7

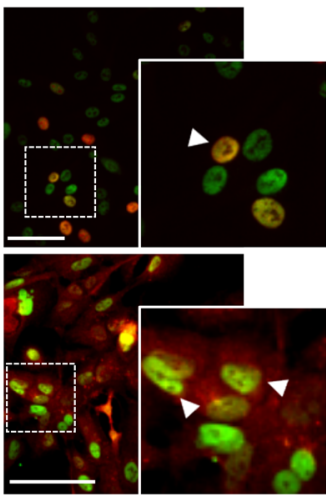

E Human melanoma cells

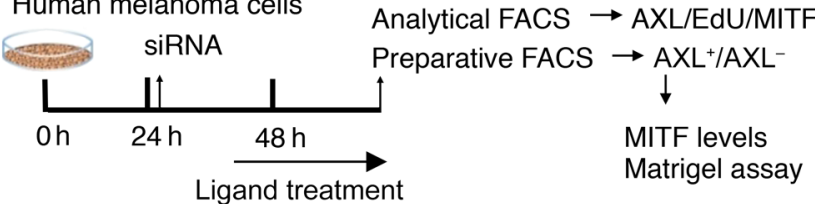

SiSMAD7

C

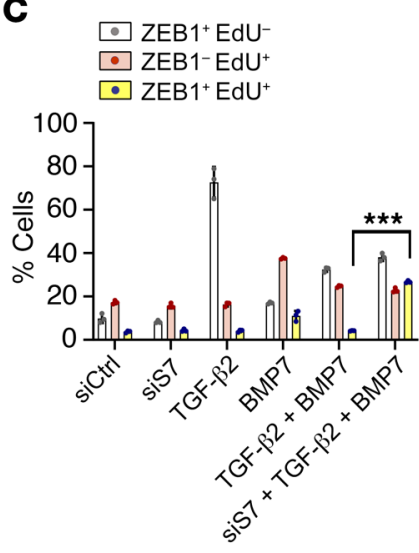

D
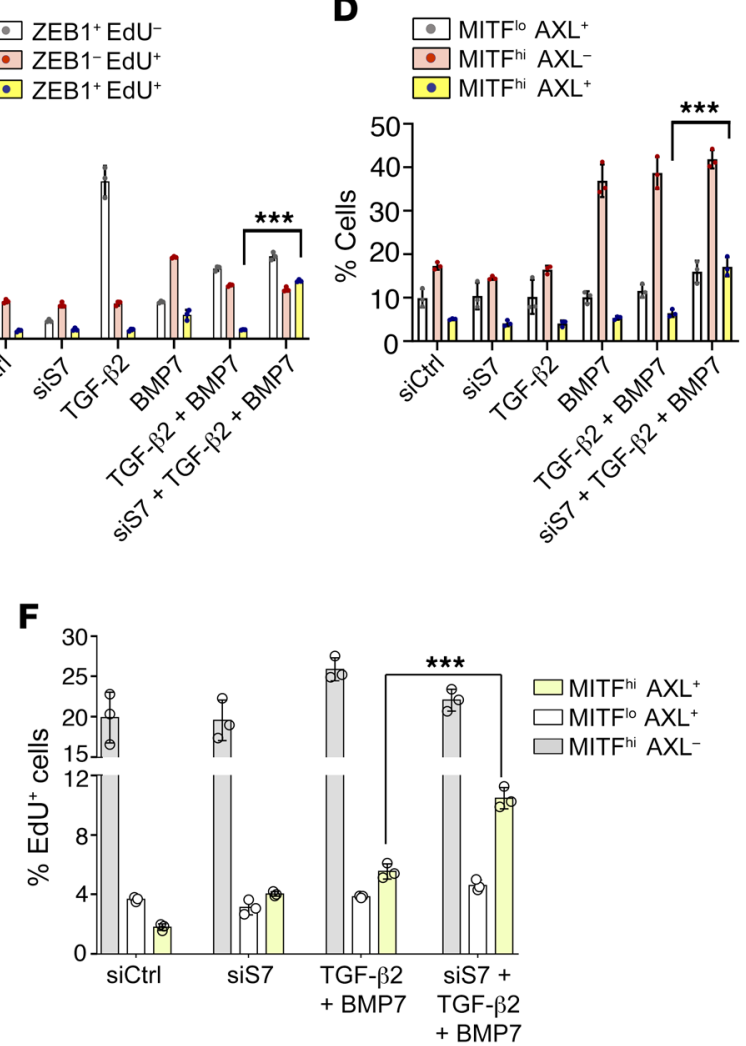

J

G

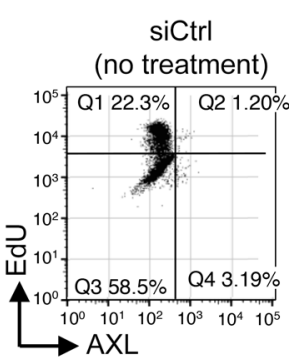

H

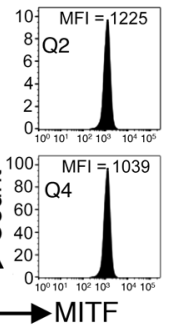

I

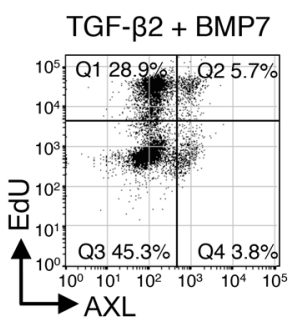

K SISMAD7

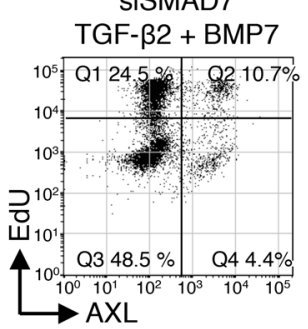

L

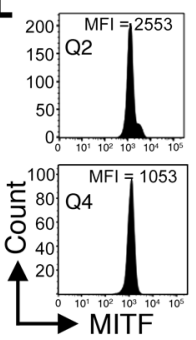

M

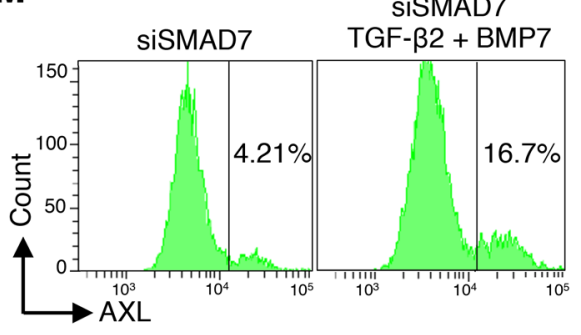

N

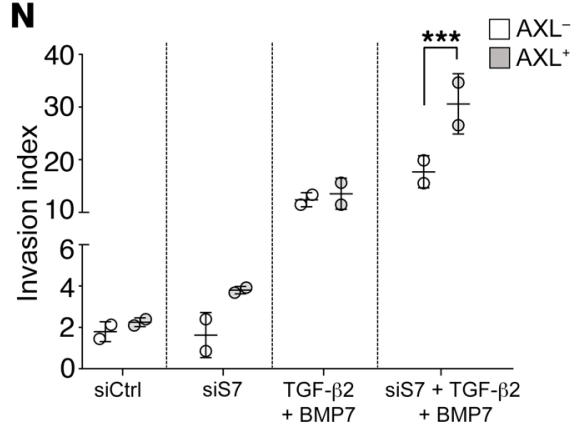

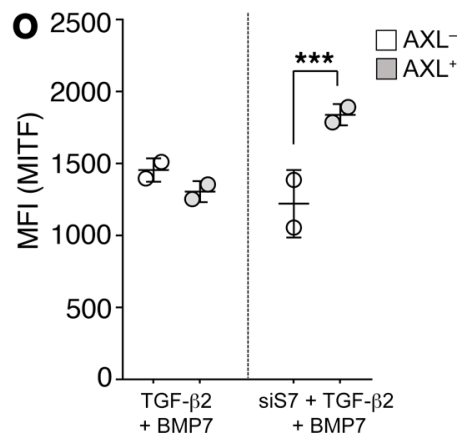

Figure 6. Loss of SMAD7 promotes emergence of proliferative-invasive MITF ${ }^{\text {hi }}$ AXL ${ }^{\text {hi }}$ melanoma cells in vitro. (A) Experimental design used in this study. Proliferative M010817 cells were exposed to combinatorial ligand treatment over 3 days. Cells were pulsed with EdU, followed by immunofluorescent staining (B) Representative IF images. White arrowheads mark ZEB1+EdU ${ }^{+}$or MITFhi $A X L^{+}$cells. Open arrowheads mark ZEB1-EdU ${ }^{+}$or MITF ${ }^{\text {hi }} A \mathrm{AL}^{-}$cells. (C) Quantification of single- and double-labeled cells for ZEB1 and EdU $(n=3)$. (D) Quantification of cells expressing AXL, MITFhi, or both $(n=3)$. (E) Experimental design and sorting approach for functional analysis of MITF ${ }^{\text {hi }} A \mathrm{AXL}^{+}$cells. (F) Quantification of EdU ${ }^{+}$proliferative cells expressing MITF ${ }^{\text {hi }} A X L$ and expressing MITF ${ }^{10} \mathrm{AXL}$ by FACS $(n=3)$. (G, I, K) Enrichment for the AXL'EdU+ subpopulation in BMP7/TGF- $\beta 2 /$ siSMAD7-treated cells as shown by FACS $(n=3)$ (yellow quadrant [Q2)] AXL+EdU+, gray quadrant [Q4] AXL+EdU-). (H, J, L) Histograms show the MFI values for MITF expression in AXL+EdU' versus AXL'EdU- cells. (M) FACS analysis of cells stained with an AXL antibody following TCF- $\beta 2$ and BMP7 treatment with or without siRNA-mediated SMAD7 knockdown. (N) Matrigel assays of sorted $A X L^{+}$and $A X L^{-}$cells. (0) MITF MFI of $A X L^{+}$and $A X L^{-}$cells following combinatorial treatment. Mean of 3 (C, $\mathbf{D}$, F) or 2 independent experiments ( $\mathbf{N}$ and $\mathbf{0}) \pm$ SD. ${ }^{* *} P<0.001$, unpaired Student's $t$ test (C, D, F, N, 0). Scale bars: $50 \mu \mathrm{m}$. 
A

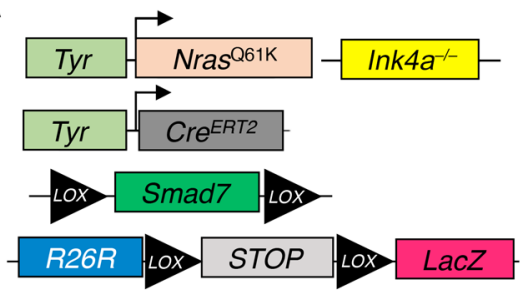

B

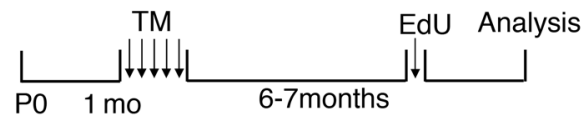

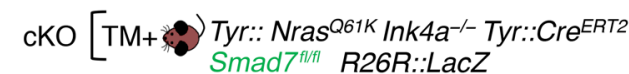

C

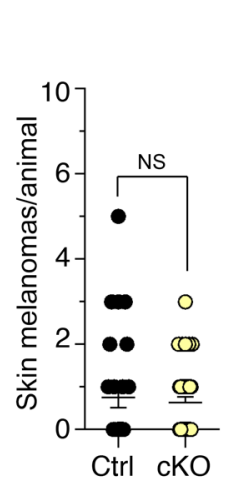

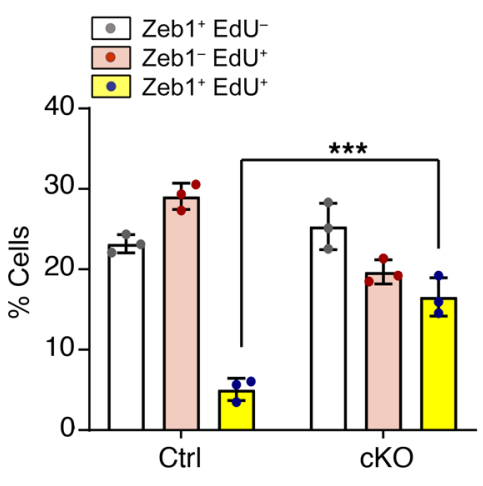

E

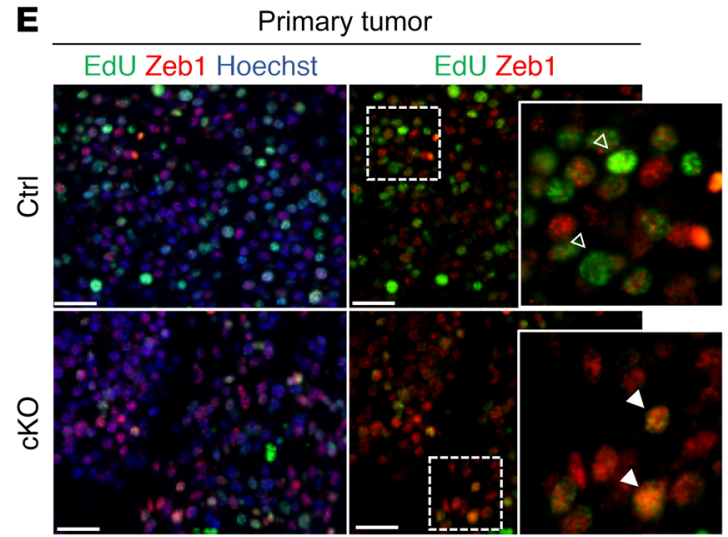

$\mathbf{F}$

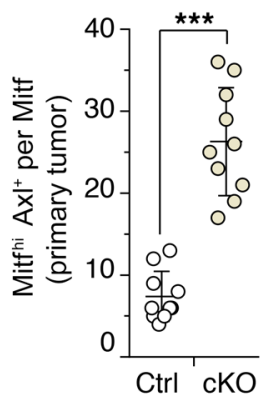

G

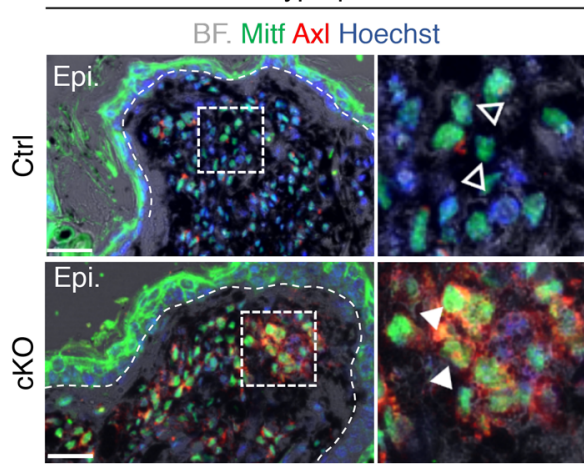

H

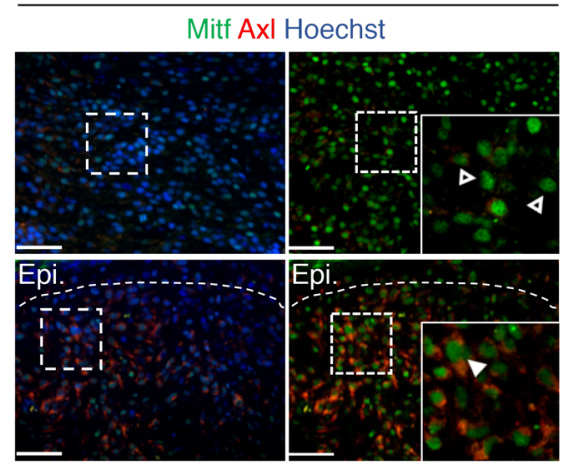

Figure 7. Loss of Smad7 promotes emergence of proliferative-invasive MITF ${ }^{\text {hi }}$ XXL $^{\text {hi }}$ melanoma cells in vivo. (A) Schematic of the melanoma mouse model used to analyze the effect of Smad7 conditional deletion in Tyr::Cre ${ }^{E R T 2}$ Tyr::Nras ${ }^{061 K} I N K 4 a^{-1-} R 26 R-L a c Z$ mice. (B) Experimental strategy used to analyze the proliferation rate by EdU injection upon Smad7 loss. (C) Quantification of the number of skin melanomas of control and Smad7-cKO mice $(n=12)$. ( $\mathbf{D}$ and $\mathbf{E})$ Immunostaining and quantification of EdU+Zeb1+ cells in Smad7-cKO and control primary tumors $(n=3)$. Gray and white bars indicate single Zeb1+ and EdU ${ }^{+}$cells, respectively. Yellow bars represents percentages of EdU+Zeb1+ cells. White arrowheads in the images indicate EdU+Zeb1+ cells, open arrowheads EdU'Zeb1 ${ }^{-}$cells. (F-H) Quantification and immunostaining of Mitf ${ }^{\text {hi }} \mathrm{Axl}^{+}$cells in $\mathrm{CKO}$ and control primary tumors. Percentages of Mitf ${ }^{\text {hi }}$ $\mathrm{Axl}^{+}$cells calculated over total number of Mitfhi and Mitflo cells $(n=10)$. Yellow bars represent percentages of Mitf ${ }^{\text {hi }}$ cells that are Axl'. White arrowheads indicate Mitfhi $\mathrm{Axl}^{+}$cells, open arrowheads Mitfhi $\mathrm{Axl}^{-}$cells. Data are represented as the mean $\pm \mathrm{SEM} .{ }^{* *} P<0.001$, unpaired Student's $t$ test (C-F). Epi, epidermis. Scale bars: $50 \mu \mathrm{m}$.

untreated, exposed to various combinations of TGF- $\beta$ superfamily factors, or treated with siSMAD7 in the absence of exogenous TGF- $\beta$ factors, AXL-positive cells were MITF $^{\mathrm{lo}}$, and only a minor fraction of cells expressed both AXL and high levels of MITF. In contrast, upon concomitant BMP7/TGF- $\beta 2 /$ siSMAD7 treatment, the number of cells double positive for AXL and high MITF was significantly increased to $18 \% \pm 3 \%$.

To determine the biological properties of this $\mathrm{MITF}^{\mathrm{hi}} \mathrm{AXL}^{\text {hi }}$ cell subpopulation, we first assessed the rate of EdU incorporation and MITF expression levels in FACS-separated AXL-posi- tive versus AXL-negative cells obtained from different culture conditions (Figure 6E and Supplemental Table 5). In control and siSmad7-treated cultures as well as in the presence of TGF- $\beta 2$ and BMP7, the majority of EdU-positive cells did not express AXL (Figure 6, F, G, I). In contrast, cultures with concomitant BMP7/ TGF- $\beta 2 /$ siSMAD7 treatment showed a significant increase in the percentage of EdU-positive proliferating cells expressing AXL (Figure 6, F and K). Moreover, MITF MFI was higher in AXL/ EdU double-positive cells as compared with AXL-positive/EdUnegative cells (Figure 6, H, J, and L, and Supplemental Table 6). 

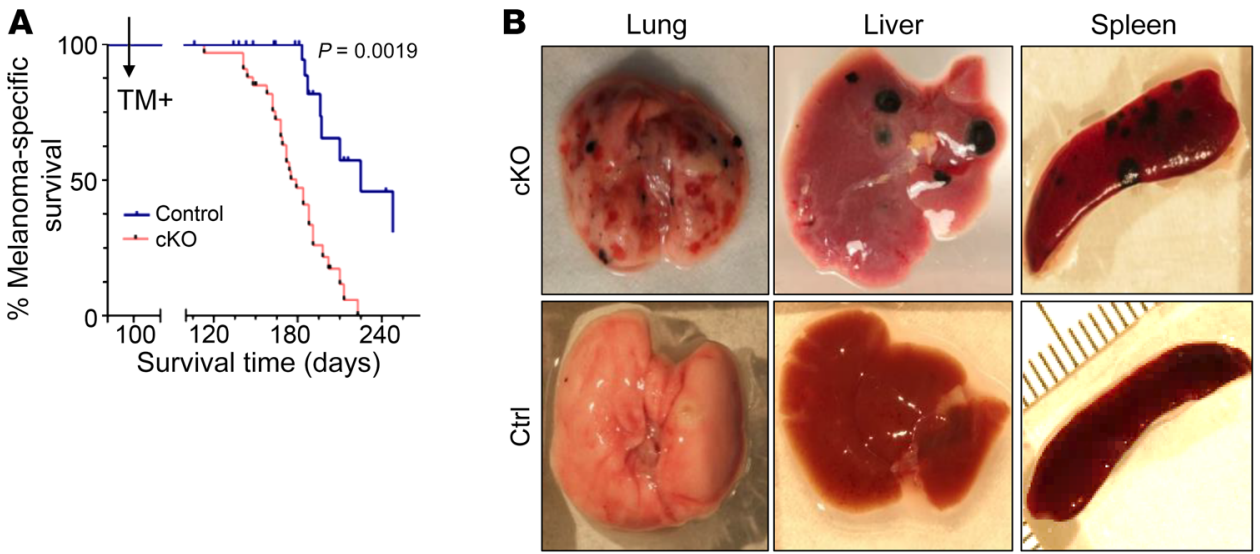

C

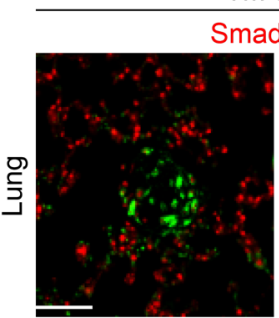

TM cKO
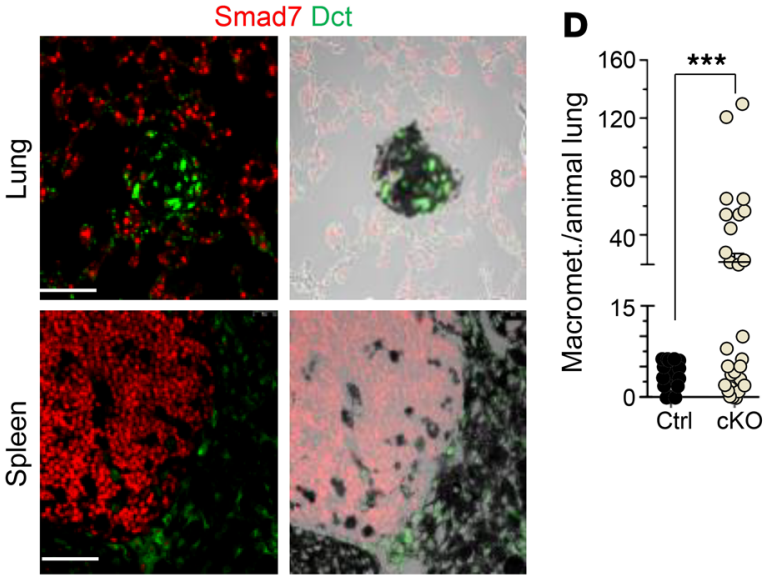

E

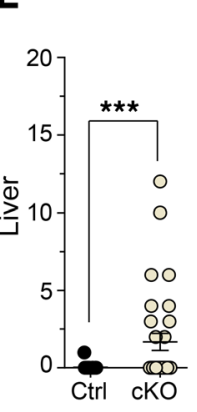

F

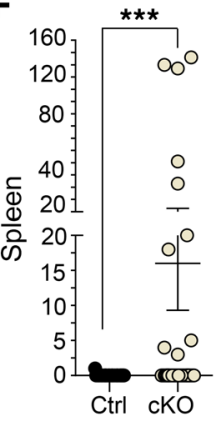

Figure 8. Reduced Smad7 expression promotes metastatic spread of melanoma in vivo. (A) Kaplan-Meier curves comparing melanoma-specific survival of control and Smad7-cKO animals ( $n$ = $35 \mathrm{cKO}, n=30$ control). (B) Representative photographs of indicated organ from Smad7-cKO and control mice. (C) Immunofluorescent staining for Smad7 and Dct at the sites of distant metastasis in Smad7-cKO and control mice. Note absence of Smad7 expression in pigmented Dct $^{+}$areas. (D-F) Quantification of macrometastasis in lung, spleen, and liver in Smad7-cKO and control littermates (lung, $n=21 \mathrm{cKO}, n=31$ control; liver, $n=24 \mathrm{cKO}, n=12$ control; spleen, cKO $n=32$, control $n=25$ ). (G) Experimental strategy used to analyze the effect of local Smad7 loss in Tyr:: $\mathrm{N}-\mathrm{Ras}^{0661 K}$ Ink4a- Tyr::Cre ${ }^{E R T 2}$ R26R::LacZ mice by 4-OHT administration to the skin. (H) Quantification of lung macrometastasis, in which Smad7 has been locally deleted in the skin of 3-week-old mice ( $n=11 \mathrm{cKO}, n=12$ control). Data are represented as a mean of 3 independent experiments $\pm \mathrm{SEM}$. ${ }^{* *} P<0.01 ;{ }^{* * *} P<$ 0.001, unpaired Student's $t$ test (D-F) or log-rank Mantel-Cox test (A). met, metastasis. Scale bar: $50 \mu \mathrm{m}$.
G

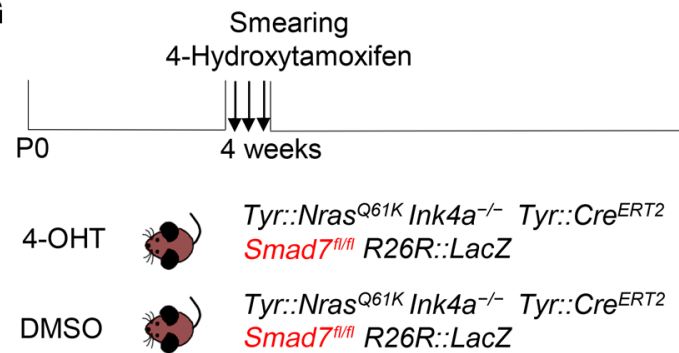

Remarkably, the highest MITF expression levels were detected in AXL/EdU double-positive cells upon silencing of SMAD7 in BMP7/TGF- $\beta 2$-treated cultures (Figure 6L).

To study the invasive potential of MITF ${ }^{\text {hi }} \mathrm{AXL}^{\text {hi }}$ cells, AXLpositive and AXL-negative cell populations were FACS isolated from human melanoma cell lines exposed to the above-mentioned conditions, followed by a Matrigel Transwell invasion assay (Figure 6, $\mathrm{E}$ and $\mathrm{M})$. Under most conditions analyzed, the invasive capacities of AXL-positive and AXL-negative cells were not significantly different. Strikingly, however, the AXL-positive population present in BMP7/TGF- $\beta 2 /$ siSMAD7-treated cultures displayed the highest invasiveness among all cell populations analyzed (Figure $6 \mathrm{~N})$. Moreover, MITF levels were markedly increased in these AXL-positive cells when compared with the other cell populations (Figure 6O). Thus, reducing SMAD7 levels in the presence of combinatorial BMP7/TGF- $\beta 2$ treatment leads to the appearance of

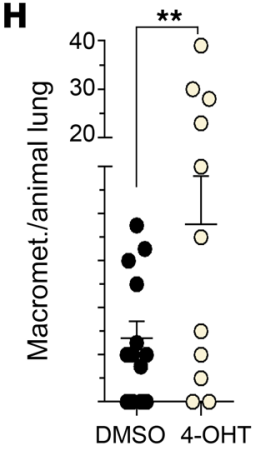

proliferative MITF ${ }^{\text {hi }} \mathrm{AXL}^{\mathrm{hi}}$ cells with invasive capacity, which possibly contributes to melanoma cell aggressiveness.

Loss of Smad7 in vivo increases the number of cells with both invasive and proliferative features. Our cell culture data revealed the presence of cells that acquired both invasive and proliferative features upon SMAD7 inactivation. To corroborate these findings in an in vivo setting, we used Nras ${ }^{661 K} \mathrm{In} 4 \mathrm{a}^{-/}$mice that also carried a floxed allele of $\operatorname{Smad7}(48)$, the Tyr::Cre ${ }^{E R T 2}$ transgene, and the R26R-LSL-LacZ reporter gene (Figure 7, A and B). TM-mediated Cre-activation at 1 month of age resulted in efficient recombination and depletion of Smad7 protein in Dct-expressing melanoma cells (Supplemental Figure 7, A-C). Importantly, loss of Smad7 led to a substantial increase in the percentage of Dct-positive melanocytic cells coexpressing nuclear pSmad2/3 and pSmad1/5/8, demonstrating activation of both canonical SMAD signaling pathways (Supplemental Figure 7, D and E). Despite signal acti- 
vation, however, deletion of Smad7 neither had an overt effect on hyperplastic dermal lesions (data not shown) nor on the number of skin melanomas emerging during disease progression (Figure 7C). Consistent with this, EdU incorporation was not significantly altered in Smad7-cKO tumors as compared with tumors of control mice ( $34 \% \pm 1 \%$ vs. $37 \% \pm 3 \%$ ) (Figure 7D). Next, we assessed Zeb1 expression in primary tumors of $\mathrm{Nras}^{\mathrm{Q} 61 \mathrm{~K}} \mathrm{Ink} 4 \mathrm{a}^{-/-}$mice after EdU application. Intriguingly, the number of Zeb1/EdU double-positive cells was significantly higher $(16 \% \pm 4 \%)$ in primary tumors of Smad7-cKO mice when compared with control primary tumors $(4 \% \pm 2 \%$ ) (Figure $7, \mathrm{D}$ and $\mathrm{E}$ ). In addition, we quantified the numbers of Mitf ${ }^{\text {hi }} \mathrm{Axl}$ double-positive cells in skin sections of Smad7-cKO mice. Hyperplastic lesions of Smad7-cKO mice displayed significantly higher numbers of double-positive cells $(10 \%$ $\pm 2 \%)$ compared with the control ( $2 \% \pm 1 \%)$. Likewise, in Smad7cKO primary tumors, the number of Mitf ${ }^{\text {hi }}$ Axl double-positive cells reached $27 \% \pm 3 \%$, as compared with $6 \% \pm 1 \%$ in control primary tumors (Figure 7, F-H). Thus, inactivation of Smad7 and a concomitant increase in $\mathrm{pSmad} 2 / 3$ and $\mathrm{pSmad} 1 / 5 / 8$ do not interfere with tumor growth in vivo, but boost the appearance of proliferating cells expressing the invasive marker Zeb1 and of Mitfli $\mathrm{Axl}^{\text {hi }}$ cells in melanocytic lesions.

Reduced Smad7 expression promotes massive metastatic spread of melanoma in vivo. Although Smad7-cKO mice apparently displayed unaltered tumor initiation and primary tumor growth, their melanoma-free survival was significantly reduced (Figure 8A). In fact, they showed a massive metastatic spread to lung, spleen, and liver, organs that often are devoid of metastases in tumor-bearing control mice (Figure 8B). Pigmented Dct-positive cells in these metastases did not express $S m a d 7$, indicating their origin from Smad7-depleted recombined melanoma cells (Figure 8C and Supplemental Figure 7C). Quantification of the metastatic burden in lung, spleen, and liver confirmed the high aggressiveness of melanoma caused by Smad7 inactivation (Figure 8, D-F).

In the above experiment, Smad7 inactivation was broadly induced in melanocytic cells by systemic TM treatment. To assess whether local depletion of Smad7 is sufficient to promote melanoma metastasis formation, we applied 4-hydroxytamoxifen (4-OHT) to the back skin of Tyr::Nras ${ }^{\mathrm{Q} 61 \mathrm{~K}}$ Ink $4 \mathrm{a}^{-1-}$ Tyr::Cre $\mathrm{ERT2}^{\mathrm{ER}}$ $S m a d 7^{f / f l}$ R26R::LacZ mice (Figure 8G). Although recombination efficiency in the treated areas was lower than in melanocytic skin cells after systemic TM treatment (Supplemental Figure 7, B and C), a reduction in overall survival of mice was observed (Supplemental Figure 7F). Importantly, at the day of sacrifice, the number of lung macrometastases was highly increased upon local Smad7 depletion, demonstrating the striking metastatic potential of Smad7-cKO skin melanoma cells (Figure 8H). Thus, loss of Smad7 significantly increases the aggressiveness of melanoma in the context of enhanced $\mathrm{pSmad} 2 / 3$ and $\mathrm{pSmad} 1 / 5 / 8$ signal activation.

\section{Discussion}

Phenotype switching, i.e., the reversible switch from a proliferative $\mathrm{MITF}^{\text {hi }} \mathrm{AXL}^{\text {lo }}$ melanoma cell to an invasive $\mathrm{MITF}^{\mathrm{lo}} \mathrm{AXL}^{\text {hi }}$ cell, is thought to represent a key mechanism underlying melanoma progression $(2,3,5,14,49)$. In the present study, however, we demonstrate that phenotype switching is not obligatory for invasion and metastatic spread of melanoma cells and that invasive melanoma cells can overcome restrictions in proliferation by intrinsically modulating their response to combinatorial TGF- $\beta$ superfamily signaling. Despite the vast literature on TGF- $\beta$ eliciting an antiproliferative and proinvasive effect in melanoma cells $(31,50,51)$, we reveal that Smad4-mediated overall canonical TGF- $\beta$ signaling is required for tumor initiation and primarily promotes, rather than antagonizes, melanoma cell proliferation in vivo. Consistent with these data, we identify BMP7 as a factor stimulating melanoma cell proliferation and able to override the antiproliferative and proinvasive activity of TGF- $\beta$ and NODAL. However, invasiveness can be triggered in cells exposed to combinatorial TGF- $\beta$ signaling by lowering levels of the inhibitory SMAD protein SMAD7. Strikingly, activation of integrated TGF- $\beta$ signaling by SMAD7 inhibition results in the emergence of an invasive program characterized by high AXL and ZEB1 expression in cells maintaining high MITF expression and proliferative capacity. Consequently, the increase in the number of Mitf ${ }^{\text {hi }}{ }^{-} x^{\text {hi }}$ cells in Smad7-depleted melanoma is associated with massive metastasis formation in vivo. Our findings demonstrate that proliferation is compatible with increased invasiveness and continued expression of MITF, revealing a mechanism that underpins melanoma aggressiveness.

Our data are compatible with earlier studies suggesting that melanoma progression can occur in the absence of phenotype switching. Recently, single-cell RNA sequencing revealed the presence of a small subpopulation of MITF ${ }^{\text {hi }} \mathrm{AXL}^{\text {hi }}$ cells in every human tumor sample assessed, although MITF and AXL expression were exclusive on the level of the bulk tumor (15). Also, a considerable fraction of circulating tumor cells isolated from invasive melanoma patients was MITF positive (52). Widmer and colleagues showed that, in cultures of melanoma cells, hypoxia could drive invasion without affecting overall proliferation (53). Furthermore, cells in invasive cell clusters did not alter MITF expression during a process referred to as cooperative invasion (54). Finally, time-lapse microscopy in vitro and ex vivo demonstrated that invading melanoma cells in culture do not necessarily undergo cell cycle arrest and actively proliferate regardless of their MITF expression status (55). Our study now reveals how a cellular state of simultaneous proliferation and invasion can be achieved by modulation of combinatorial TGF- $\beta$ signaling in melanoma cells. Conceivably, changes in overall TGF- $\beta$ signal activity and thus emergence of invasive-proliferative $\mathrm{MITF}^{\text {hi }} \mathrm{AXL}^{\text {hi }}$ cells during melanoma progression are dependent on the composition of growth factors, the availability of nutrients and/or oxygen in the microenvironment, and notably, the regulation of downstream signaling molecules such as SMAD7.

It is well established that the biological effects of TGF- $\beta$ superfamily signaling are context dependent, but how specific responses to TGF- $\beta$ family factors are controlled is still largely unknown (23). In melanoma, several TGF- $\beta$ superfamily factors, including TGF- $\beta$, ACTIVINA, NODAL, BMP2, BMP4, and BMP7, are known to be expressed and to activate signaling in an autocrine manner (27, 41, 56-58). However, BMP7 promoted proliferation, whereas BMP4 induced cell cycle arrest in melanoma cells at concentrations at which both factors activated the pSMAD1/5/8-branch of TGF- $\beta$ signaling to a comparable extent. In agreement with other studies $(6,42,59)$, cell cycle arrest was also induced upon treatment with either TGF- $\beta$ or NODAL, which, unlike BMP4, act 
through pSMAD2/3 rather than pSMAD1/5/8. Of note, however, nuclear pSMAD2 $/ 3$ activity is not only present in TGF- $\beta$-treated arrested cells, but also readily detectable in proliferative melanoma cells in vitro and in vivo $(6,31,32)$. Furthermore, loss of SMAD7 enhanced both pSMAD1/5/8 and pSMAD2/3 levels in melanoma cell cultures and in our melanoma mouse model, but predominantly boosted TGF- $\beta$ /NODAL-induced invasiveness rather than BMP7-induced proliferation. Thus, the phenotype obtained upon reducing SMAD7 in the context of combinatorial TGF- $\beta$ superfamily signaling cannot simply be explained by preferential usage of either pSMAD2/3 or pSMAD1/5/8 signaling.

Our finding that BMP7 acts as a proproliferative factor in melanoma even in the presence of TGF- $\beta / \mathrm{pSMAD} 2 / 3$ signaling is in line with studies in systems other than melanoma, in which BMP7 was reported to override effects of TGF- $\beta$. For instance, TGF- $\beta$ / NODAL and BMP7 counterregulate each other during pathophysiological processes in various organs, such as lung, liver, and kidney (60-62). In breast cancer cells, BMP7 inhibits expression of TGF- $\beta$ activated genes associated with EMT, resulting in a significant reduction in TGF- $\beta$-triggered cell migration and invasiveness in culture (63), reminiscent of our findings in melanoma. It remains to be shown whether in these cases the balance between integrated BMP7- and TGF- $\beta$-dependent signaling outcome can be tipped by modulation of SMAD7 activity in a manner similar to what we observed in melanoma, where an EMT gene expression signature, loss of cell-substrate adhesion in vitro, invasiveness, and metastasis formation in vivo were all promoted upon SMAD7 inactivation in spite of the presence of BMP7 and pSMAD1/5/8 activity.

Our experiments indicate that overall canonical TGF- $\beta$ / SMAD signaling is a potent promoter of melanoma progression already at early stages of the disease. Unlike depletion of TGFbRII, which interferes with pSMAD2-mediated signaling and results in hair graying associated with disturbed melanocyte stem cell maintenance (64), eliminating the common downstream signaling mediator SMAD4 did not interfere with normal melanocyte survival and proliferation. These findings suggest that TGFbRII and $S m a d 4$ inactivation differentially affect the finely tuned balance of combined signaling by BMP and TGF- $\beta$ ligands in the bulge niche $(65,66)$. Importantly, our data also demonstrate that the inhibitory effect of Smad4-cKO on Nras ${ }^{\mathrm{Q} 61 \mathrm{~K}}$-driven hyperpigmentation and tumor initiation observed in our mouse melanoma model cannot simply be due to depletion of the melanocytic lineage. Indeed, taking advantage of an inducible system allowing gene manipulation at various stages of tumor progression, we show that Smad4 is required for proliferation in established skin melanoma. This, in turn, reveals the existence of and necessity for proproliferative activators of the Smad pathway, such as BMP7, in the context of melanoma propagation. Since proproliferative BMP7 appears to be dominant over proinvasive factors, the question arises of how a metastatic process is induced in melanoma. One possible mechanism might involve spatiotemporal changes in the composition of the TGF- $\beta$ superfamily factors to which a tumor cell is exposed, where reducing levels of proproliferative factors relative to proinvasive factor concentrations would promote the development of invasive cells with limited proliferation capacity (8). Whether such phenotype switching indeed occurs in vivo remains to be shown. Our study offers an alternative, but nonexclusive, mechanism of metastasis formation, which involves the reduction of SMAD7 levels in the presence of proproliferative and proinvasive TGF- $\beta$ superfamily factors. Although it is currently unknown how SMAD7 expression is normally regulated in melanoma, targeting SMAD7 level-dependent emergence of proliferative cells with metastatic capacity might represent a powerful treatment strategy, possibly in combination with drugs interfering with tumor cell proliferation. Indeed, high levels of ZEB1 expression, which we show to be induced by SMAD7 inactivation, have been associated with inherent resistance to MAPKi in $B R A F^{V 600 E}$-mutant melanoma (46). Likewise, a shift toward $\mathrm{AXL}^{\text {hi }}$ expression was observed in melanoma samples resistant to RAF and MEK inhibition (12, 14, 15). Importantly, our study provides a system for investigating how tumor cell-intrinsic changes independent of phenotype switching contribute to malignant progression of melanoma.

\section{Methods}

All detailed information on experimental procedures and reagents is provided in Supplemental Tables 8, 9, and 10 and Supplemental Experimental Procedures.

Mice. All implementations were carried out in Tyr::Nras ${ }^{\mathrm{Q} 61 \mathrm{~K}} \mathrm{Ink} 4 \mathrm{a}^{-/-}$ Tyr::Cre ${ }^{E R T 2}$ R26R::LacZ animals kept on a mixed genetic background.

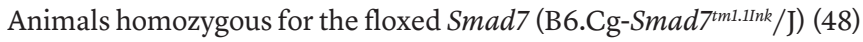
allele or the floxed Smad4 allele (Smad4 $4^{\text {tm2.1Cxd } / J) ~(67) ~ w e r e ~ m a t e d ~}$ with mice heterozygous for the respective floxed allele or alleles and carrying the TM-inducible Cre ${ }^{E R T 2}$ recombinase under the control of a Tyrosinase promoter (mice provided by L. Chin, The University of Texas MD Anderson Cancer Center, Houston, Texas, USA). Genotyping was done by PCR on genomic DNA (QIAGEN Taq PCR Core Kit 201225) using the primer pairs indicated in Supplemental Table 7. DNA was prepared, and the reactions were carried out as described $(48,67)$. The presence of the various alleles did not change the expected sex, and Mendelian inheritance ratio was found. Mice were examined frequently and sacrificed at an end point defined by adverse clinical symptoms, such as multiple skin tumors (diameter $>5 \mathrm{~mm}$ ), weight loss (>15\%), or hunched posture.

Data and software availability. All RNA-Seq data were deposited in the NCBI's Gene Expression Omnibus database (GEO GSE113472).

Statistics. All data were reported either as mean \pm SEM or mean \pm SD. Results were evaluated using the statistical tests indicated in the figure legends. $P$ values and the number of independent experiments are indicated in figure legends. $P<0.05$ was considered statistically significant. Survival curves and metastasis were investigated by Kaplan-Meier analysis (Unistat) of $\mathrm{H}$-score, which were compared by univariate and multivariate Cox regression (Stata). Quantifications of immunofluorescent staining were done on sections of at least 3 different mice. For each animal, 3 different sections and, for each section, 300-500 label-positive cells were counted.

Study approval. Primary human materials were provided by the Tumor Biopsy and the Live Cell Biobanks of the University Research Priority Program (URPP) Translational Cancer Research (Mitchell P. Levesque, University Hospital Zurich). Biobanking of surplus, human material from consenting melanoma patients was performed according to the Declaration of Helsinki on Human Rights and was approved by the IRB of Zurich (EK.647/800). All research on surplus human material was conducted under IRB approval KEK-Zh.Nr 2014-0425. To ensure patient confidentiality, no information on age, gender, or 
ethnicity was provided to the investigators of this study. All studies involving mice were approved by the veterinary authorities of the Canton of Zurich, Switzerland, and were performed in accordance with Swiss law regarding welfare and treatment of animals.

\section{Author contributions}

LS, DZ, ET, and RRC, conceived the project and designed the research. ET, PC, RRC, and SNF performed experiments and analyzed data. DZ, CXD, SV, MPL, RD, and IK provided analytic tools and intellectual contributions to the design of experiments and interpretation of data. LS and ET wrote the original manuscript. All authors discussed the results and commented on the manuscript.

\section{Acknowledgments}

We thank Heinz Arnheiter for critical reading of the manuscript; Claudia Meyer and Astrid Rhyner for assistance in histology;
Raphaël Bossart and Jessica Häusel for help with genotyping; the Center for Microscopy and Image Analysis at the University of Zurich for help with slide scanning; and the center of flow cytometry for help with sorting. We thank F. Beermann, L. Chin, and M. Serrano for providing Tyr::Nras ${ }^{Q 61 K}$ Ink $4 a^{-/-}$, Tyr::Cre ${ }^{E R T 2}$, and R26R-LSL-LacZ mice. We acknowledge the advice by the Cancer Biology PhD Program of the University of Zurich. This work was supported by the Swiss National Science Foundation (31003A_169859), the Swiss Cancer League (KFS-3682-08-2015), and the University Research Priority Program (URPP) Translational Cancer Research.

Address correspondence to: Lukas Sommer, Stem Cell Biology, Institute of Anatomy, University of Zurich, Winterthurerstrasse 190, CH-8057 Zurich, Switzerland. Phone: 41.44.63.55443; Email: lukas.sommer@anatomy.uzh.ch.
1. Siegel RL, Miller KD, Jemal A. Cancer statistics, 2016. CA Cancer JClin. 2016;66(1):7-30.

2. Goding CR. Commentary. A picture of Mitf in melanoma immortality. Oncogene. 2011;30(20):2304-2306.

3. Cheli Y, et al. Mitf is the key molecular switch between mouse or human melanoma initiating cells and their differentiated progeny. Oncogene. 2011;30(20):2307-2318.

4. Falletta $P$, et al. Translation reprogramming is an evolutionarily conserved driver of phenotypic plasticity and therapeutic resistance in melanoma. Genes Dev. 2017;31(1):18-33.

5. Restivo $G$, et al. The low affinity neurotrophin receptor CD271 regulates phenotype switching in melanoma. Nat Commun. 2017;8(1):1988.

6. Schlegel NC, von Planta A, Widmer DS, Dummer $\mathrm{R}$, Christofori G. PI3K signalling is required for a TGF $\beta$-induced epithelial-mesenchymal-like transition (EMT-like) in human melanoma cells. Exp Dermatol. 2015;24(1):22-28.

7. Hoek KS, et al. In vivo switching of human melanoma cells between proliferative and invasive states. Cancer Res. 2008;68(3):650-656.

8. Hölzel M, Tüting T. Inflammation-induced plasticity in melanoma therapy and metastasis. Trends Immunol. 2016;37(6):364-374.

9. Garraway LA, et al. Integrative genomic analyses identify MITF as a lineage survival oncogene amplified in malignant melanoma. Nature. 2005;436(7047):117-122.

10. Chen $\mathrm{X}$, et al. Integration of external signaling pathways with the core transcriptional network in embryonic stem cells. Cell. 2008;133(6):1106-1117.

11. Wu X, Liu X, Koul S, Lee CY, Zhang Z, Halmos B. AXL kinase as a novel target for cancer therapy. Oncotarget. 2014;5(20):9546-9563.

12. Boshuizen J, et al. Cooperative targeting of melanoma heterogeneity with an AXL antibody-drug conjugate and BRAF/MEK inhibitors. Nat Med. 2018;24(2):203-212.

13. Carreira S, et al. Mitf regulation of Dia1 controls melanoma proliferation and invasiveness. Genes Dev. 2006;20(24):3426-3439.

14. Müller J, et al. Low MITF/AXL ratio predicts early resistance to multiple targeted drugs in melanoma. Nat Commun. 2014;5:5712.
15. Tirosh I, et al. Dissecting the multicellular ecosystem of metastatic melanoma by single-cell RNA-Seq. Science. 2016;352(6282):189-196.

16. Yao Z, et al. TGF-beta IL-6 axis mediates selective and adaptive mechanisms of resistance to molecular targeted therapy in lung cancer. Proc Natl Acad Sci USA. 2010;107(35):15535-15540.

17. Thomson S, Petti F, Sujka-Kwok I, Epstein D, Haley JD. Kinase switching in mesenchymal-like non-small cell lung cancer lines contributes to EGFR inhibitor resistance through pathway redundancy. Clin Exp Metastasis. 2008;25(8):843-854.

18. Valastyan S, Weinberg RA. Tumor metastasis: molecular insights and evolving paradigms. Cell. 2011;147(2):275-292.

19. Lamouille S, Xu J, Derynck R. Molecular mechanisms of epithelial-mesenchymal transition. Nat Rev Mol Cell Biol. 2014;15(3):178-196.

20. Vandamme N, Berx G. Melanoma cells revive an embryonic transcriptional network to dictate phenotypic heterogeneity. Front Oncol. 2014;4:352.

21. Caramel J, et al. A switch in the expression of embryonic EMT-inducers drives the development of malignant melanoma. Cancer Cell. 2013;24(4):466-480.

22. Javelaud D, Alexaki VI, Mauviel A. Transforming growth factor-beta in cutaneous melanoma. Pigment Cell Melanoma Res. 2008;21(2):123-132.

23. Massagué J. TGF $\beta$ signalling in context. Nat Rev Mol Cell Biol. 2012;13(10):616-630.

24. Gregory PA, et al. An autocrine TGF-beta/ZEB/ miR-200 signaling network regulates establishment and maintenance of epithelial-mesenchymal transition. Mol Biol Cell. 2011;22(10):1686-1698.

25. Fang R, et al. Nodal promotes aggressive phenotype via Snail-mediated epithelial-mesenchymal transition in murine melanoma. Cancer Lett. 2013;333(1):66-75.

26. Rothhammer T, Bataille F, Spruss T, Eissner G, Bosserhoff AK. Functional implication of BMP4 expression on angiogenesis in malignant melanoma. Oncogene. 2007;26(28):4158-4170.

27. Topczewska JM, et al. Embryonic and tumorigenic pathways converge via Nodal signaling: role in melanoma aggressiveness. Nat Med.
2006;12(8):925-932.

28. Yan X, Liu Z, Chen Y. Regulation of TGF-beta signaling by Smad7. Acta Biochim Biophys Sin (Shanghai). 2009;41(4):263-272.

29. Javelaud D, et al. Stable overexpression of Smad7 in human melanoma cells impairs bone metastasis. Cancer Res. 2007;67(5):2317-2324.

30. Mohammad KS, et al. TGF-beta-RI kinase inhibitor SD-208 reduces the development and progression of melanoma bone metastases. Cancer Res. 2011;71(1):175-184.

31. Rodeck U, Nishiyama T, Mauviel A. Independent regulation of growth and SMAD-mediated transcription by transforming growth factor beta in human melanoma cells. Cancer Res. 1999;59(3):547-550.

32. Lo RS, Witte ON. Transforming growth factorbeta activation promotes genetic contextdependent invasion of immortalized melanocytes. Cancer Res. 2008;68(11):4248-4257.

33. Goumans MJ, et al. Activin receptor-like kinase (ALK) 1 is an antagonistic mediator of lateral TGFbeta/ALK5 signaling. Mol Cell. 2003;12(4):817-828.

34. Liu F, et al. A human Mad protein acting as a BMP-regulated transcriptional activator. Nature. 1996;381(6583):620-623.

35. Schmierer B, Hill CS. TGFbeta-SMAD signal transduction: molecular specificity and functional flexibility. Nat Rev Mol Cell Biol. 2007;8(12):970-982.

36. Ackermann J, Frutschi M, Kaloulis K, McKee T, Trumpp A, Beermann F. Metastasizing melanoma formation caused by expression of activated $\mathrm{N}$-RasQ61K on an INK4a-deficient background. Cancer Res. 2005;65(10):4005-4011.

37. Zingg D, et al. The epigenetic modifier EZH2 controls melanoma growth and metastasis through silencing of distinct tumour suppressors. Nat Commun. 2015;6:6051.

38. Siegel PM, Massagué J. Cytostatic and apoptotic actions of TGF-beta in homeostasis and cancer. Nat Rev Cancer. 2003;3(11):807-821.

39. Krasagakis K, et al. Desensitization of melanoma cells to autocrine TGF-beta isoforms. J Cell Physiol. 1999;178(2):179-187.

40. Rothhammer T, Poser I, Soncin F, Bataille F, 
Moser M, Bosserhoff AK. Bone morphogenic proteins are overexpressed in malignant melanoma and promote cell invasion and migration. Cancer Res. 2005;65(2):448-456.

41. Stove C, Vanrobaeys F, Devreese B, Van Beeumen J, Mareel M, Bracke M. Melanoma cells secrete follistatin, an antagonist of activin-mediated growth inhibition. Oncogene. 2004;23(31):5330-5339.

42. Schlegel NC, Eichhoff OM, Hemmi S, Werner S, Dummer R, Hoek KS. Id 2 suppression of p 15 counters TGF-beta-mediated growth inhibition of melanoma cells. Pigment Cell Melanoma Res. 2009;22(4):445-453.

43. Cancer Genome Atlas Network. Genomic classification of cutaneous melanoma. Cell. 2015;161(7):1681-1696.

44. Bindea G, et al. ClueGO: a Cytoscape plug-in to decipher functionally grouped gene ontology and pathway annotation networks. Bioinformatics. 2009;25(8):1091-1093.

45. Verfaillie A, et al. Decoding the regulatory landscape of melanoma reveals TEADS as regulators of the invasive cell state. Nat Commun. 2015;6:6683.

46. Richard G, et al. ZEB1-mediated melanoma cell plasticity enhances resistance to MAPK inhibitors. EMBO Mol Med. 2016;8(10):1143-1161.

47. Smith MP, et al. Effect of SMURF2 targeting on susceptibility to MEK inhibitors in melanoma. J Natl Cancer Inst. 2013;105(1):33-46.

48. Kleiter I, et al. Smad7 in T cells drives T helper 1 responses in multiple sclerosis and experimental autoimmune encephalomyelitis. Brain. 2010;133(Pt 4):1067-1081.

49. Hoek KS, Goding CR. Cancer stem cells versus phenotype-switching in melanoma. Pigment Cell Melanoma Res. 2010;23(6):746-759.
50. Janji B, Melchior C, Gouon V, Vallar L, Kieffer N. Autocrine TGF-beta-regulated expression of adhesion receptors and integrin-linked kinase in HT-144 melanoma cells correlates with their metastatic phenotype. Int J Cancer. 1999;83(2):255-262.

51. Miyoshi E, et al. Transforming growth factor beta up-regulates expression of the $\mathrm{N}$-acetylglucosaminyltransferase $\mathrm{V}$ gene in mouse melanoma cells. J Biol Chem. 1995;270(11):6216-6220.

52. Khoja L, et al. Prevalence and heterogeneity of circulating tumour cells in metastatic cutaneous melanoma. Melanoma Res. 2014;24(1):40-46.

53. Widmer DS, et al. Hypoxia contributes to melanoma heterogeneity by triggering HIF $1 \alpha-$ dependent phenotype switching. J Invest Dermatol. 2013;133(10):2436-2443.

54. Chapman A, Fernandez del Ama L, Ferguson J, Kamarashev J, Wellbrock C, Hurlstone A. Heterogeneous tumor subpopulations cooperate to drive invasion. Cell Rep. 2014;8(3):688-695.

55. Haass NK, et al. Real-time cell cycle imaging during melanoma growth, invasion, and drug response. Pigment Cell Melanoma Res. 2014;27(5):764-776.

56. Hsu MY, Rovinsky S, Penmatcha S, Herlyn M, Muirhead D. Bone morphogenetic proteins in melanoma: angel or devil? Cancer Metastasis Rev . 2005;24(2):251-263.

57. Perrot CY, Javelaud D, Mauviel A. Insights into the transforming growth factor- $\beta$ signaling pathway in cutaneous melanoma. Ann Dermatol. 2013;25(2):135-144.

58. Lázár-Molnár E, Hegyesi H, Tóth S, Falus A. Autocrine and paracrine regulation by cytokines and growth factors in melanoma. Cytokine. 2000;12(6):547-554.
59. Hendrix MJ, Seftor EA, Seftor RE, KasemeierKulesa J, Kulesa PM, Postovit LM. Reprogramming metastatic tumour cells with embryonic microenvironments. Nat Rev Cancer. 2007;7(4):246-255.

60. Pegorier S, Campbell GA, Kay AB, Lloyd CM. Bone morphogenetic protein (BMP)- 4 and BMP-7 regulate differentially transforming growth factor (TGF)-beta1 in normal human lung fibroblasts (NHLF). Respir Res. 2010;11:85.

61. Zeisberg M, et al. BMP-7 counteracts TGFbeta1-induced epithelial-to-mesenchymal transition and reverses chronic renal injury. Nat Med. 2003;9(7):964-968.

62. Zeisberg M, et al. Fibroblasts derive from hepatocytes in liver fibrosis via epithelial to mesenchymal transition.J Biol Chem. 2007;282(32):23337-23347.

63. Ying X, Sun Y, He P. Bone morphogenetic protein-7 inhibits EMT-associated genes in breast cancer. Cell Physiol Biochem. 2015;37(4):1271-1278.

64. Nishimura EK, et al. Key roles for transforming growth factor beta in melanocyte stem cell maintenance. Cell Stem Cell. 2010;6(2):130-140.

65. Oshimori N, Fuchs E. Paracrine TGF- $\beta$ signaling counterbalances BMP-mediated repression in hair follicle stem cell activation. Cell Stem Cell. 2012;10(1):63-75.

66. Sharov AA, et al. Bone morphogenetic protein (BMP) signaling controls hair pigmentation by means of cross-talk with the melanocortin receptor-1 pathway. Proc Natl Acad Sci U S A. 2005;102(1):93-98.

67. Yang X, Li C, Herrera PL, Deng CX. Generation of Smad4/Dpc4 conditional knockout mice. Genesis. 2002;32(2):80-81. 